\title{
Generalized Mixed Equilibria, Variational Inclusions, and Fixed Point Problems
}

\author{
A. E. Al-Mazrooei, ${ }^{1}$ A. S. M. Alofi, ${ }^{1}$ A. Latif, ${ }^{1}$ and J.-C. Yao ${ }^{1,2}$ \\ ${ }^{1}$ Department of Mathematics, King Abdulaziz University, P.O. Box 80203, Jeddah 21589, Saudi Arabia \\ ${ }^{2}$ Center for Fundamental Science, Kaohsiung Medical University, Kaohsiung 807, Taiwan \\ Correspondence should be addressed to A. Latif; alatif@kau.edu.sa
}

Received 3 April 2014; Accepted 19 May 2014; Published 12 June 2014

Academic Editor: Chong Li

Copyright (C) 2014 A. E. Al-Mazrooei et al. This is an open access article distributed under the Creative Commons Attribution License, which permits unrestricted use, distribution, and reproduction in any medium, provided the original work is properly cited.

\begin{abstract}
We propose two iterative algorithms for finding a common element of the set of solutions of finite generalized mixed equilibrium problems, the set of solutions of finite variational inclusions for maximal monotone and inverse strong monotone mappings, and the set of common fixed points of infinite nonexpansive mappings and an asymptotically $\kappa$-strict pseudocontractive mapping in the intermediate sense in a real Hilbert space. We prove some strong and weak convergence theorems for the proposed iterative algorithms under suitable conditions.
\end{abstract}

\section{Introduction}

Let $H$ be a real Hilbert space with inner product $\langle\cdot, \cdot\rangle$ and norm $\|\cdot\|$, let $C$ be a nonempty closed convex subset of $H$, and let $P_{C}$ be the metric projection of $H$ onto $C$. Let $S: C \rightarrow C$ be a self-mapping on $C$. $\operatorname{Fix}(S)$ is the set of fixed points of $S$ and $\mathbf{R}$ is the set of all real numbers. We recall that a mapping $A: C \rightarrow H$ is said to be $L$-Lipschitz continuous, if there exists a constant $L \geq 0$ such that

$$
\|A x-A y\| \leq L\|x-y\|, \quad \forall x, y \in C .
$$

If $L=1$, then $A$ is called a nonexpansive mapping and if $L \epsilon$ $[0,1)$, then $A$ is called a contraction. We also let $I$ denote the identity operator on the Hilbert space $H$.

Let $\varphi: C \rightarrow \mathbf{R}$ be a real-valued function, let $A:$ $C \rightarrow H$ be a nonlinear mapping, and let $F: C \times C \rightarrow \mathbf{R}$ be a bifunction. The generalized mixed equilibrium problem (GMEP) introduced in [1] is to find $x \in C$ such that

$$
F(x, y)+\varphi(y)-\varphi(x)+\langle A x, y-x\rangle \geq 0, \quad \forall y \in C .
$$

We denote the set of solutions of GMEP (2) by $\operatorname{GMEP}(F, \varphi, A)$. The GMEP covers many problems [2$6]$ as special cases and has been extensively studied recently.

Throughout this paper, we assume as in [1] that $F: C \times$ $C \rightarrow \mathbf{R}$ is a bifunction satisfying conditions (A1)-(A4) and $\varphi: C \rightarrow \mathbf{R}$ is a lower semicontinuous and convex function with restriction (B1) or (B2), where

(A1) $F(x, x)=0$, for all $x \in C$;

(A2) $F$ is monotone; that is, $F(x, y)+F(y, x) \leq 0$, for any $x, y \in C$;

(A3) $F$ is upper-hemicontinuous; that is, for each $x, y, z \in$ C,

$$
\limsup _{t \rightarrow 0^{+}} F(t z+(1-t) x, y) \leq F(x, y) ;
$$

(A4) $F(x, \cdot)$ is convex and lower semicontinuous, for each $x \in C$;

(B1) for each $x \in H$ and $r>0$, there exists a bounded subset $D_{x} \subset C$ and $y_{x} \in C$ such that, for any $z \in$ $C \backslash D_{x}$,

$$
F\left(z, y_{x}\right)+\varphi\left(y_{x}\right)-\varphi(z)+\frac{1}{r}\left\langle y_{x}-z, z-x\right\rangle<0 ;
$$

(B2) $C$ is a bounded set.

Next we list some elementary consequences for the mixed equilibrium problem studied in [2] where $\operatorname{MEP}(F, \varphi)$ is the solution set. 
Proposition 1 (see [2]). Assume that $F: C \times C \rightarrow \mathbf{R}$ satisfies (A1)-(A4) and let $\varphi: C \rightarrow \mathbf{R}$ be a proper lower semicontinuous and convex function. Assume that either (B1) or (B2) holds. For $r>0$ and $x \in H$, define a mapping $T_{r}^{(F, \varphi)}: H \rightarrow C$ as follows:

$$
\begin{aligned}
T_{r}^{(F, \varphi)}(x)=\{z & \in C: F(z, y)+\varphi(y)-\varphi(z) \\
& \left.+\frac{1}{r}\langle y-z, z-x\rangle \geq 0, \forall y \in C\right\},
\end{aligned}
$$

for all $x \in H$. Then the following hold:

(i) for each $x \in H, T_{r}^{(F, \varphi)}(x) \neq \emptyset$;

(ii) $T_{r}^{(F, \varphi)}$ is single-valued;

(iii) $T_{r}^{(F, \varphi)}$ is firmly nonexpansive; that is, for any $x, y \in H$,

$$
\left\|T_{r}^{(F, \varphi)} x-T_{r}^{(F, \varphi)} y\right\|^{2} \leq\left\langle T_{r}^{(F, \varphi)} x-T_{r}^{(F, \varphi)} y, x-y\right\rangle ;
$$

(iv) $\operatorname{Fix}\left(T_{r}^{(F, \varphi)}\right)=\operatorname{MEP}(F, \varphi)$;

(v) $\operatorname{MEP}(F, \varphi)$ is closed and convex.

Next, recall some concepts.

Definition 2. Let $C$ be a nonempty subset of a normed space $X$ and let $S: C \rightarrow C$ be a self-mapping on $C$.

(i) $S$ is asymptotically nonexpansive (see [7]), if there exists a sequence $\left\{k_{n}\right\}$ of positive numbers satisfying the property $\lim _{n \rightarrow \infty} k_{n}=1$ and

$$
\left\|S^{n} x-S^{n} y\right\| \leq k_{n}\|x-y\|, \quad \forall n \geq 1, \forall x, y \in C .
$$

(ii) $S$ is asymptotically nonexpansive in the intermediate sense (see [8]) provided that $S$ is uniformly continuous and

$$
\limsup _{n \rightarrow \infty} \sup _{x, y \in C}\left(\left\|S^{n} x-S^{n} y\right\|-\|x-y\|\right) \leq 0 .
$$

(iii) $S$ is uniformly Lipschitzian, if there exists a constant $\mathscr{L}>0$ such that

$$
\left\|S^{n} x-S^{n} y\right\| \leq \mathscr{L}\|x-y\|, \quad \forall n \geq 1, \forall x, y \in C .
$$

It is clear that every nonexpansive mapping is asymptotically nonexpansive and every asymptotically nonexpansive mapping is uniformly Lipschitzian. Recently, Kim and Xu [9] introduced the concept of asymptotically $\kappa$-strict pseudocontractive mappings in a Hilbert space as follows.

Definition 3. Let $C$ be a nonempty subset of a Hilbert space $H$. A mapping $S: C \rightarrow C$ is said to be an asymptotically $\kappa$ strict pseudocontractive mapping with sequence $\left\{\gamma_{n}\right\}$, if there exist a constant $\kappa \in[0,1)$ and a sequence $\left\{\gamma_{n}\right\}$ in $[0, \infty)$ with $\lim _{n \rightarrow \infty} \gamma_{n}=0$ such that

$$
\left\|S^{n} x-S^{n} y\right\|^{2} \leq\left(1+\gamma_{n}\right)\|x-y\|^{2}+\kappa\left\|x-S^{n} x-\left(y-S^{n} y\right)\right\|^{2},
$$

$\forall n \geq 1, \quad \forall x, y \in C$.
They studied weak and strong convergence theorems for this class of mappings. It is important to note that every asymptotically $\mathcal{\kappa}$-strict pseudocontractive mapping with sequence $\left\{\gamma_{n}\right\}$ is a uniformly $\mathscr{L}$-Lipschitzian mapping with $\mathscr{L}=\sup \left\{\left(\kappa+\sqrt{1+(1-\kappa) \gamma_{n}}\right) /(1+\kappa): n \geq 1\right\}$.

Recently, Sahu et al. [10] considered the concept of asymptotically $\kappa$-strict pseudocontractive mappings in the intermediate sense, which are not necessarily Lipschitzian.

Definition 4. Let $C$ be a nonempty subset of a Hilbert space $H$. A mapping $S: C \rightarrow C$ is said to be an asymptotically $\kappa$ strict pseudocontractive mapping in the intermediate sense with sequence $\left\{\gamma_{n}\right\}$, if there exist a constant $\kappa \in[0,1)$ and a sequence $\left\{\gamma_{n}\right\}$ in $[0, \infty)$ with $\lim _{n \rightarrow \infty} \gamma_{n}=0$ such that

$$
\begin{array}{r}
\limsup _{n \rightarrow \infty} \sup _{x, y \in C}\left(\left\|S^{n} x-S^{n} y\right\|^{2}-\left(1+\gamma_{n}\right)\|x-y\|^{2}\right. \\
\left.-\kappa\left\|x-S^{n} x-\left(y-S^{n} y\right)\right\|^{2}\right) \leq 0 .
\end{array}
$$

Put $c_{n}:=\max \left\{0, \sup _{x, y \in C}\left(\left\|S^{n} x-S^{n} y\right\|^{2}-\left(1+\gamma_{n}\right)\|x-y\|^{2}-\right.\right.$ $\left.\left.\kappa\left\|x-S^{n} x-\left(y-S^{n} y\right)\right\|^{2}\right)\right\}$. Then, $c_{n} \geq 0(\forall n \geq 1), c_{n} \rightarrow$ $0(n \rightarrow \infty)$, and (11) reduces to the relation

$$
\begin{aligned}
\left\|S^{n} x-S^{n} y\right\|^{2} \leq & \left(1+\gamma_{n}\right)\|x-y\|^{2}+\kappa\left\|x-S^{n} x-\left(y-S^{n} y\right)\right\|^{2} \\
& +c_{n}, \quad \forall n \geq 1, \forall x, y \in C .
\end{aligned}
$$

Whenever $c_{n}=0$, for all $n \geq 1$ in (12), then $S$ is an asymptotically $\kappa$-strict pseudocontractive mapping with sequence $\left\{\gamma_{n}\right\}$.

Let $B$ be a single-valued mapping of $C$ into $H$ and $R$ be a multivalued mapping with domain $D(R)=C$. Consider the following variational inclusion: find a point $x \in C$ such that

$$
0 \in B x+R x
$$

We denote by $I(B, R)$ the solution set of the variational inclusion (13). In 1998, Huang [11] studied problem (13) in the case where $R$ is maximal monotone and $B$ is strongly monotone and Lipschitz continuous with $D(R)=C=H$. Subsequently, Zeng et al. [12] further studied problem (13) in the case which is more general than that in [11]. Moreover, the authors [12] obtained the same strong convergence conclusion as in [11]. In addition, the authors also gave the geometric convergence rate estimate for approximate solutions.

In this paper, inspired by the research work mentioned above, we introduce two iterative algorithms for finding a common element of the set of solutions of finite generalized mixed equilibrium problems, the set of solutions of finite variational inclusions for maximal monotone and inverse strong monotone mappings, and the set of common fixed points of infinite nonexpansive mappings and an asymptotically $\kappa$ strict pseudocontractive mapping in the intermediate sense in a real Hilbert space. We prove some strong and weak convergence theorems for the proposed iterative algorithms under mild conditions. 


\section{Preliminaries}

Let $H$ be a real Hilbert space and $C$ be a nonempty closed convex subset of $H$. We use the notation $x_{n} \rightarrow x$ to indicate that the sequence $\left\{x_{n}\right\}$ converges weakly to $x$ and $x_{n} \rightarrow x$ to indicate that the sequence $\left\{x_{n}\right\}$ converges strongly to $x$. Moreover, we use $\omega_{w}\left(x_{n}\right)$ to denote the weak $\omega$-limit set of the sequence $\left\{x_{n}\right\}$; that is,

$\omega_{w}\left(x_{n}\right)$

$:=\left\{x \in H: x_{n_{i}} \rightarrow x\right.$ for some subsequence $\left\{x_{n_{i}}\right\}$ of $\left.\left\{x_{n}\right\}\right\}$.

Recall that a mapping $A: C \rightarrow H$ is called

(i) Monotone, if

$$
\langle A x-A y, x-y\rangle \geq 0, \quad \forall x, y \in C ;
$$

(ii) $\eta$-strongly monotone, if there exists a constant $\eta>0$ such that

$$
\langle A x-A y, x-y\rangle \geq \eta\|x-y\|^{2}, \quad \forall x, y \in C
$$

(iii) $\alpha$-inverse strongly monotone, if there exists a constant $\alpha>0$ such that

$$
\langle A x-A y, x-y\rangle \geq \alpha\|A x-A y\|^{2}, \quad \forall x, y \in C .
$$

When $A$ is an $\alpha$-inverse strongly monotone mapping of $C$ into $H$, it is easy to see that $A$ is $(1 / \alpha)$-Lipschitz continuous. We also have that, for all $u, v \in C$ and $\lambda>0$,

$$
\begin{aligned}
\|(I-\lambda A) u-(I-\lambda A) v\|^{2} \\
\quad=\|(u-v)-\lambda(A u-A v)\|^{2} \\
\quad=\|u-v\|^{2}-2 \lambda\langle A u-A v, u-v\rangle+\lambda^{2}\|A u-A v\|^{2} \\
\quad \leq\|u-v\|^{2}+\lambda(\lambda-2 \alpha)\|A u-A v\|^{2} .
\end{aligned}
$$

So, if $\lambda \leq 2 \alpha$, then $I-\lambda A$ is a nonexpansive mapping from $C$ to $H$.

The metric projection from $H$ onto $C$ is the mapping $P_{C}$ : $H \rightarrow C$ which assigns to each point $x \in H$ the unique point $P_{C} x \in C$ satisfying the property

$$
\left\|x-P_{C} x\right\|=\inf _{y \in C}\|x-y\|=: d(x, C) .
$$

Some important properties of projections are listed in the following proposition.

Proposition 5. For given $x \in H$ and $z \in C$,

(i) $z=P_{C} x \Leftrightarrow\langle x-z, y-z\rangle \leq 0, \forall y \in C$;

(ii) $z=P_{C} x \Leftrightarrow\|x-z\|^{2} \leq\|x-y\|^{2}-\|y-z\|^{2}, \forall y \in C$;

(iii) $\left\langle P_{C} x-P_{C} y, x-y\right\rangle \geq\left\|P_{C} x-P_{C} y\right\|^{2}, \forall y \in H$.

Consequently, $P_{C}$ is nonexpansive and monotone. It is easy to see that the projection $P_{C}$ is 1-ism.
We need some facts and tools in a real Hilbert space $H$ which are listed as lemmas below.

Lemma 6. Let $X$ be a real inner product space. Then there holds the following inequality:

$$
\|x+y\|^{2} \leq\|x\|^{2}+2\langle y, x+y\rangle, \quad \forall x, y \in X .
$$

Lemma 7. Let $H$ be a real Hilbert space. Then the following hold:

(a) $\|x-y\|^{2}=\|x\|^{2}-\|y\|^{2}-2\langle x-y, y\rangle$, for all $x, y \in H$;

(b) $\|\lambda x+\mu y+\nu z\|^{2}=\lambda\|x\|^{2}+\mu\|y\|^{2}+\nu\|z\|^{2}-$ $\lambda \mu\|x-y\|^{2}-\mu \nu\|y-z\|^{2}-\lambda \nu\|x-z\|^{2}$, for all $x, y, z \in$ $H$ and $\lambda, \mu, \nu \in[0,1]$ with $\lambda+\mu+\nu=1$;

(c) if $\left\{x_{n}\right\}$ is a sequence in $H$ such that $x_{n} \rightarrow x$, it follows that

$\limsup _{n \rightarrow \infty}\left\|x_{n}-y\right\|^{2}=\limsup _{n \rightarrow \infty}\left\|x_{n}-x\right\|^{2}+\|x-y\|^{2}, \quad \forall y \in H$.

Lemma 8 (see [10, Lemma 2.5]). Let $H$ be a real Hilbert space. Given a nonempty closed convex subset $C$ of $H$ and points $x, y, z \in H$ and given also a real number $a \in \mathbf{R}$, the set

$$
\left\{v \in C:\|y-v\|^{2} \leq\|x-v\|^{2}+\langle z, v\rangle+a\right\}
$$

is convex and closed.

Lemma 9 (see [10, Lemma 2.6]). Let $C$ be a nonempty subset of a Hilbert space $H$ and let $S: C \rightarrow C$ be an asymptotically $\kappa$-strict pseudocontractive mapping in the intermediate sense with sequence $\left\{\gamma_{n}\right\}$. Then

$$
\begin{aligned}
& \left\|S^{n} x-S^{n} y\right\| \\
& \leq \frac{1}{1-\kappa} \\
& \quad \times\left(\kappa\|x-y\|+\sqrt{\left(1+(1-\kappa) \gamma_{n}\right)\|x-y\|^{2}+(1-\kappa) c_{n}}\right),
\end{aligned}
$$

for all $x, y \in C$ and $n \geq 1$.

Lemma 10 (see [10, Lemma 2.7]). Let $C$ be a nonempty subset of a Hilbert space $H$ and let $S: C \rightarrow C$ be a uniformly continuous asymptotically $\kappa$-strict pseudocontractive mapping in the intermediate sense with sequence $\left\{\gamma_{n}\right\}$. Let $\left\{x_{n}\right\}$ be a sequence in $C$ such that $\left\|x_{n}-x_{n+1}\right\| \rightarrow 0$ and $\left\|x_{n}-S^{n} x_{n}\right\| \rightarrow 0$ as $n \rightarrow \infty$. Then $\left\|x_{n}-S x_{n}\right\| \rightarrow 0$ as $n \rightarrow \infty$.

Lemma 11 (demiclosedness principle [10, Proposition 3.1]). Let $C$ be a nonempty closed convex subset of a Hilbert space $H$ and let $S: C \rightarrow C$ be a continuous asymptotically $\kappa$-strict pseudocontractive mapping in the intermediate sense with sequence $\left\{\gamma_{n}\right\}$. Then $I-S$ is demiclosed at zero in the sense that if $\left\{x_{n}\right\}$ is a sequence in $C$ such that $x_{n} \rightarrow x \in C$ and $\lim \sup _{m \rightarrow \infty} \lim \sup _{n \rightarrow \infty}\left\|x_{n}-S^{m} x_{n}\right\|=0$, then $(I-S) x=0$. 
Lemma 12 (see [10, Proposition 3.2]). Let $C$ be a nonempty closed convex subset of a Hilbert space $H$ and let $S: C \rightarrow$ $C$ be a continuous asymptotically $\kappa$-strict pseudocontractive mapping in the intermediate sense with sequence $\left\{\gamma_{n}\right\}$ such that $\operatorname{Fix}(S) \neq \emptyset$. Then, $\operatorname{Fix}(S)$ is closed and convex.

Lemma 13 (see [13, page 80]). Let $\left\{a_{n}\right\}_{n=1}^{\infty},\left\{b_{n}\right\}_{n=1}^{\infty}$, and $\left\{\delta_{n}\right\}_{n=1}^{\infty}$ be sequences of nonnegative real numbers satisfying the inequality

$$
a_{n+1} \leq\left(1+\delta_{n}\right) a_{n}+b_{n}, \quad \forall n \geq 1
$$

If $\sum_{n=1}^{\infty} \delta_{n}<\infty$ and $\sum_{n=1}^{\infty} b_{n}<\infty$, then $\lim _{n \rightarrow \infty} a_{n}$ exists. If, in addition, $\left\{a_{n}\right\}_{n=1}^{\infty}$ has a subsequence which converges to zero, then $\lim _{n \rightarrow \infty} a_{n}=0$.

Corollary 14 (see [14, page 303]). Let $\left\{a_{n}\right\}_{n=0}^{\infty}$ and $\left\{b_{n}\right\}_{n=0}^{\infty}$ be two sequences of nonnegative real numbers satisfying the inequality

$$
a_{n+1} \leq a_{n}+b_{n}, \quad \forall n \geq 0 .
$$

If $\sum_{n=0}^{\infty} b_{n}$ converges, then $\lim _{n \rightarrow \infty} a_{n}$ exists.

Recall that a Banach space $X$ is said to satisfy the Opial condition [15], if for any given sequence $\left\{x_{n}\right\} \subset X$ which converges weakly to an element $x \in X$, there holds the inequality

$$
\limsup _{n \rightarrow \infty}\left\|x_{n}-x\right\|<\limsup _{n \rightarrow \infty}\left\|x_{n}-y\right\|, \quad \forall y \in X, y \neq x
$$

It is well known in [15] that every Hilbert space $H$ satisfies the Opial condition.

Lemma 15 (see [16, Proposition 3.1]). Let $C$ be a nonempty closed convex subset of a real Hilbert space $H$ and let $\left\{x_{n}\right\}$ be a sequence in $H$. Suppose that

$$
\left\|x_{n+1}-p\right\|^{2} \leq\left(1+\lambda_{n}\right)\left\|x_{n}-p\right\|^{2}+\delta_{n}, \quad \forall p \in C, n \geq 1,
$$

where $\left\{\lambda_{n}\right\}$ and $\left\{\delta_{n}\right\}$ are sequences of nonnegative real numbers such that $\sum_{n=1}^{\infty} \lambda_{n}<\infty$ and $\sum_{n=1}^{\infty} \delta_{n}<\infty$. Then $\left\{P_{C} x_{n}\right\}$ converges strongly in $C$.

Lemma 16 (see [17]). Let $C$ be a closed convex subset of a real Hilbert space $H$. Let $\left\{x_{n}\right\}$ be a sequence in $H$ and $u \in H$. Let $q=P_{C} u$. If $\left\{x_{n}\right\}$ is such that $\omega_{w}\left(x_{n}\right) \subset C$ and satisfies the condition

$$
\left\|x_{n}-u\right\| \leq\|u-q\|, \quad \forall n
$$

then $x_{n} \rightarrow$ qas $n \rightarrow \infty$.

Let $\left\{T_{n}\right\}_{n=1}^{\infty}$ be an infinite family of nonexpansive selfmappings on $C$ and let $\left\{\lambda_{n}\right\}_{n=1}^{\infty}$ be a sequence of nonnegative numbers in $[0,1]$. For any $n \geq 1$, define a self-mapping $W_{n}$ on $C$ as follows:

$$
\begin{aligned}
U_{n, n+1} & =I, \\
U_{n, n} & =\lambda_{n} T_{n} U_{n, n+1}+\left(1-\lambda_{n}\right) I, \\
U_{n, n-1} & =\lambda_{n-1} T_{n-1} U_{n, n}+\left(1-\lambda_{n-1}\right) I, \\
& \vdots \\
U_{n, k} & =\lambda_{k} T_{k} U_{n, k+1}+\left(1-\lambda_{k}\right) I, \\
U_{n, k-1} & =\lambda_{k-1} T_{k-1} U_{n, k}+\left(1-\lambda_{k-1}\right) I, \\
& \vdots \\
U_{n, 2} & =\lambda_{2} T_{2} U_{n, 3}+\left(1-\lambda_{2}\right) I, \\
W_{n} & =U_{n, 1}=\lambda_{1} T_{1} U_{n, 2}+\left(1-\lambda_{1}\right) I .
\end{aligned}
$$

Such a mapping $W_{n}$ is called the $W$-mapping generated by $T_{n}, T_{n-1}, \ldots, T_{1}$ and $\lambda_{n}, \lambda_{n-1}, \ldots, \lambda_{1}$.

Lemma 17 (see [18, Lemma 3.2]). Let $C$ be a nonempty closed convex subset of a real Hilbert space $H$. Let $\left\{T_{n}\right\}_{n=1}^{\infty}$ be a sequence of nonexpansive self-mappings on $C$ such that $\bigcap_{n=1}^{\infty} \operatorname{Fix}\left(T_{n}\right) \neq \emptyset$ and let $\left\{\lambda_{n}\right\}$ be a sequence in $(0, b]$ for some $b \in(0,1)$. Then, for every $x \in C$ and $k \geq 1$, the limit $\lim _{n \rightarrow \infty} U_{n, k} x$ exists.

Utilizing Lemma 17, we can define a mapping $W: H \rightarrow$ $H$ as follows:

$$
W x=\lim _{n \rightarrow \infty} W_{n} x=\lim _{n \rightarrow \infty} U_{n, 1} x, \quad \forall x \in H .
$$

Such a $W$ is called the $W$-mapping generated by $T_{1}, T_{2}, \ldots$ and $\lambda_{1}, \lambda_{2}, \ldots$. We remark that since $W_{n}$ is nonexpansive, $W: H \rightarrow H$ is also nonexpansive.

Lemma 18 (see [18, Lemma 3.3]). Let $C$ be a nonempty closed convex subset of a real Hilbert space $H$. Let $\left\{T_{n}\right\}_{n=1}^{\infty}$ be a sequence of nonexpansive self-mappings on $C$ such that $\bigcap_{n=1}^{\infty} \operatorname{Fix}\left(T_{n}\right) \neq \emptyset$, and let $\left\{\lambda_{n}\right\}$ be a sequence in $(0, b]$, for some $b \in(0,1)$. Then, $\operatorname{Fix}(W)=\bigcap_{n=1}^{\infty} \operatorname{Fix}\left(T_{n}\right)$.

Lemma 19 (see [19, demiclosedness principle]). Let $C$ be a nonempty closed convex subset of a real Hilbert space $H$. Let $T$ be a nonexpansive self-mapping on $C$. Then $I-T$ is demiclosed. That is, whenever $\left\{x_{n}\right\}$ is a sequence in $C$ weakly converging to some $x \in C$ and the sequence $\left\{(I-T) x_{n}\right\}$ strongly converges to some $y$, it follows that $(I-T) x=y$.

Recall that a set-valued mapping $R: D(R) \subset H \rightarrow 2^{H}$ is called monotone, if, for all $x, y \in D(R), f \in R(x)$, and $g \in R(y)$, we have

$$
\langle f-g, x-y\rangle \geq 0 .
$$

A set-valued mapping $R$ is called maximal monotone, if $R$ is monotone and $(I+\lambda R) D(R)=H$, for each $\lambda>0$. We denote 
by $G(R)$ the graph of $R$. It is known that a monotone mapping $R$ is maximal if and only if, for $(x, f) \in H \times H,\langle f-g, x-y\rangle \geq$ 0 , for every $(y, g) \in G(R)$, we have $f \in R(x)$.

Assume that $R: D(R) \subset H \rightarrow 2^{H}$ is a maximal monotone mapping. Then, for $\lambda>0$, associated with $R$, the resolvent operator $J_{R, \lambda}$ can be defined as

$$
J_{R, \lambda} x=(I+\lambda R)^{-1} x, \quad \forall x \in H .
$$

We have the following property for the resolvent operator $J_{R, \lambda}: H \rightarrow \overline{D(R)}$.

Lemma 20 (see [11]). $J_{R, \lambda}$ is single-valued and firmly nonexpansive; that is,

$$
\left\langle J_{R, \lambda} x-J_{R, \lambda} y, x-y\right\rangle \geq\left\|J_{R, \lambda} x-J_{R, \lambda} y\right\|^{2}, \quad \forall x, y \in H .
$$

Consequently, $J_{R, \lambda}$ is nonexpansive and monotone.

Lemma 21 (see [20]). Let $R$ be a maximal monotone mapping with $D(R)=C$. Then, for any given $\lambda>0, u \in C$ is a solution of the variational inclusion: find a point $x \in C$ such that

$$
0 \in B x+R x
$$

where $B$ is a single-valued mapping of $C$ into $H$ if and only if $u \in C$ satisfies

$$
u=J_{R, \lambda}(u-\lambda B u)
$$

Lemma 22 (see [12]). Let $R$ be a maximal monotone mapping with $D(R)=C$ and let $B: C \rightarrow H$ be a strongly monotone, continuous, and single-valued mapping. Then, for each $z \in H$, the equation $z \in(B+\lambda R) x$ has a unique solution $x_{\lambda}$, for $\lambda>0$.

Lemma 23 (see [20]). Let $R$ be a maximal monotone mapping with $D(R)=C$ and let $B: C \rightarrow H$ be a monotone, continuous, and single-valued mapping. Then $(I+\lambda(R+B)) C=H$, for each $\lambda>0$. In this case, $R+B$ is maximal monotone.

\section{Strong Convergence Theorem}

In this section, we prove a strong convergence theorem for an iterative algorithm for finding a common element of the set of solutions of finite generalized mixed equilibrium problems, the set of solutions of finite variational inclusions for maximal monotone and inverse strong monotone mappings, and the set of common fixed points of infinite nonexpansive mappings and asymptotically $\kappa$-strict pseudocontractive mapping $S: C \rightarrow C$ in the intermediate sense in a real Hilbert space. This iterative algorithm is based on the extragradient method [21], Mann-type iterative method, and shrinking projection method. For more recent related results, see [22] and the references therein.

Theorem 24. Let $C$ be a nonempty closed convex subset of a real Hilbert space $H$. Let $M, N$ be two integers. Let $F_{k}$ be a bifunction from $C \times C$ to $\mathbf{R}$ satisfying (A1)-(A4) and let $\varphi_{k}: C \rightarrow \mathbf{R} \cup\{+\infty\}$ be a proper lower semicontinuous and convex function, where $k \in\{1,2, \ldots, M\}$. Let $R_{i}: C \rightarrow 2^{H}$ be a maximal monotone mapping and let $A_{k}: H \rightarrow H$ and $B_{i}: C \rightarrow H$ be $\mu_{k}$-inverse strongly monotone and $\eta_{i}$-inverse strongly monotone, respectively, where $k \in\{1,2, \ldots, M\}$ and $i \in\{1,2, \ldots, N\}$. Let $S: C \rightarrow C$ be a uniformly continuous asymptotically $\kappa$-strict pseudocontractive mapping in the intermediate sense, for some $0 \leq \kappa<1$, with sequence $\left\{\gamma_{n}\right\} \subset[0, \infty)$ such that $\lim _{n \rightarrow \infty} \gamma_{n}=0$ and $\left\{c_{n}\right\} \subset[0, \infty)$ such that $\lim _{n \rightarrow \infty} c_{n}=0$. Let $\left\{T_{n}\right\}_{n=1}^{\infty}$ be a sequence of nonexpansive self-mappings on $C$ and let $\left\{\lambda_{n}\right\}$ be a sequence in $(0, b]$ for some $b \in(0,1)$. Assume that $F:=\bigcap_{n=1}^{\infty} \operatorname{Fix}\left(T_{n}\right) \cap$ $\bigcap_{k=1}^{M} \operatorname{GMEP}\left(F_{k}, \varphi_{k}, A_{k}\right) \cap \bigcap_{i=1}^{N} I\left(B_{i}, R_{i}\right) \cap \operatorname{Fix}(S)$ is nonempty and bounded and that either (B1) or (B2) holds. Let $\left\{\alpha_{n}\right\},\left\{\beta_{n}\right\}$, and $\left\{\delta_{n}\right\}$ be sequences in $[0,1]$ such that $\alpha_{n}+\beta_{n} \leq 1,0<$ $a \leq \alpha_{n} \leq 1$, and $\kappa \leq \delta_{n} \leq d<1$. Pick any $x_{0} \in H$ and set $C_{1}=C, x_{1}=P_{C_{1}} x_{0}$. Let $\left\{x_{n}\right\}$ be a sequence generated by the following algorithm:

$$
\begin{aligned}
u_{n}= & T_{r_{M, n}}^{\left(F_{M}, \varphi_{M}\right)}\left(I-r_{M, n} A_{M}\right) T_{r_{M-1, n}}^{\left(F_{M-1}, \varphi_{M-1}\right)} \\
& \times\left(I-r_{M-1, n} A_{M-1}\right) \cdots T_{r_{1, n}}^{\left(F_{1}, \varphi_{1}\right)}\left(I-r_{1, n} A_{1}\right) x_{n}, \\
z_{n}= & J_{R_{N}, \lambda_{N, n}}\left(I-\lambda_{N, n} B_{N}\right) J_{R_{N-1}, \lambda_{N-1, n}} \\
& \times\left(I-\lambda_{N-1, n} B_{N-1}\right) \cdots J_{R_{1}, \lambda_{1, n}}\left(I-\lambda_{1, n} B_{1}\right) u_{n}, \\
k_{n}= & \delta_{n} z_{n}+\left(1-\delta_{n}\right) S^{n} z_{n}, \\
y_{n}= & \left(1-\alpha_{n}-\beta_{n}\right) x_{n}+\alpha_{n} k_{n}+\beta_{n} W_{n} z_{n}, \\
C_{n+1}= & \left\{z \in C_{n}:\left\|y_{n}-z\right\|^{2} \leq\left\|x_{n}-z\right\|^{2}+\theta_{n}\right\}, \\
x_{n+1}= & P_{C_{n+1}} x_{0}, \quad \forall n \geq 0,
\end{aligned}
$$

where $W_{n}$ is the $W$-mapping generated by (2.2), $\theta_{n}=\gamma_{n} \Delta_{n}^{2}+c_{n}$, and $\Delta_{n}=\sup \left\{\left\|x_{n}-p\right\|: p \in F\right\}<\infty$. Assume that the following conditions hold:

(i) $0<\liminf _{n \rightarrow \infty} \beta_{n} \leq \lim \sup _{n \rightarrow \infty}\left(\alpha_{n}+\beta_{n}\right)<1$;

(ii) $\left\{\lambda_{i, n}\right\} \subset\left[a_{i}, b_{i}\right] \subset\left(0,2 \eta_{i}\right), \forall i \in\{1,2, \ldots, N\}$;

(iii) $\left\{r_{k, n}\right\} \subset\left[e_{k}, f_{k}\right] \subset\left(0,2 \mu_{k}\right), \forall k \in\{1,2, \ldots, M\}$.

Then $\left\{x_{n}\right\}$ converges strongly to $P_{F} x_{0}$.

Proof. We divide the proof into several steps.

Step 1 . First note that the defining inequality in $C_{n}$ is equivalent to the inequality

$$
\left\langle 2\left(x_{n}-z_{n}\right), z\right\rangle \leq\left\|x_{n}\right\|^{2}-\left\|z_{n}\right\|^{2}+\theta_{n} .
$$

So, by Lemma $8, C_{n}$ is closed and convex, for every $n \geq 1$.

We next show that $F \subset C_{n}$, for all $n \geq 1$. Put

$$
\begin{aligned}
\Theta_{n}^{k}= & T_{r_{k, n}}^{\left(F_{k}, \varphi_{k}\right)}\left(I-r_{k, n} A_{k}\right) T_{r_{k-1, n}}^{\left(F_{k-1}, \varphi_{k-1}\right)} \\
& \times\left(I-r_{k-1, n} A_{k-1}\right) \cdots T_{r_{1, n}}^{\left(F_{1}, \varphi_{1}\right)}\left(I-r_{1, n} A_{1}\right) x_{n},
\end{aligned}
$$

for all $k \in\{1,2, \ldots, M\}$ and $n \geq 1$;

$$
\begin{aligned}
\Omega_{n}^{i}= & J_{R_{i}, \lambda_{i, n}}\left(I-\lambda_{i, n} B_{i}\right) J_{R_{i-1}, \lambda_{i-1, n}} \\
& \times\left(I-\lambda_{i-1, n} B_{i-1}\right) \cdots J_{R_{1}, \lambda_{1, n}}\left(I-\lambda_{1, n} B_{1}\right),
\end{aligned}
$$


for all $i \in\{1,2, \ldots, N\}$ and $n \geq 1$, and $\Theta_{n}^{0}=\Omega_{n}^{0}=I$, where $I$ is the identity mapping on $H$. Then we have that $u_{n}=\Theta_{n}^{M} x_{n}$ and $z_{n}=\Omega_{n}^{N} u_{n}$. Suppose that $F \subset C_{n}$ for some $n \geq 1$. Take $p \in F$ arbitrarily. Then, from (18) and Proposition 1(iii), we have

$$
\begin{aligned}
\left\|u_{n}-p\right\|= & \| T_{r_{M, n}}^{\left(F_{M,}, \varphi_{M}\right)}\left(I-r_{M, n} A_{M}\right) \Theta_{n}^{M-1} x_{n} \\
& \quad-T_{r_{M, n}}^{\left(F_{M}, \varphi_{M}\right)}\left(I-r_{M, n} A_{M}\right) \Theta_{n}^{M-1} p \| \\
\leq & \left\|\left(I-r_{M, n} A_{M}\right) \Theta_{n}^{M-1} x_{n}-\left(I-r_{M, n} A_{M}\right) \Theta_{n}^{M-1} p\right\| \\
\leq & \left\|\Theta_{n}^{M-1} x_{n}-\Theta_{n}^{M-1} p\right\| \\
& \vdots \\
\leq & \left\|\Theta_{n}^{0} x_{n}-\Theta_{n}^{0} p\right\| \\
= & \left\|x_{n}-p\right\| .
\end{aligned}
$$

Similarly, we have

$$
\begin{aligned}
\left\|z_{n}-p\right\|= & \| J_{R_{N}, \lambda_{N, n}}\left(I-\lambda_{N, n} B_{N}\right) \Omega_{n}^{N-1} u_{n} \\
& \quad-J_{R_{N}, \lambda_{N, n}}\left(I-\lambda_{N, n} B_{N}\right) \Omega_{n}^{N-1} p \| \\
\leq & \left\|\left(I-\lambda_{N, n} B_{N}\right) \Omega_{n}^{N-1} u_{n}-\left(I-\lambda_{N, n} B_{N}\right) \Omega_{n}^{N-1} p\right\| \\
\leq & \left\|\Omega_{n}^{N-1} u_{n}-\Omega_{n}^{N-1} p\right\| \\
\vdots & \\
\leq & \left\|\Omega_{n}^{0} x_{n}-\Omega_{n}^{0} p\right\| \\
= & \left\|u_{n}-p\right\| .
\end{aligned}
$$

Combining (40) and (41), we have

$$
\left\|z_{n}-p\right\| \leq\left\|x_{n}-p\right\|
$$

By Lemma 7(b), we deduce from (36) and (42) that

$$
\begin{aligned}
\left\|k_{n}-p\right\|^{2}= & \left\|\delta_{n}\left(z_{n}-p\right)+\left(1-\delta_{n}\right)\left(S^{n} z_{n}-p\right)\right\|^{2} \\
= & \delta_{n}\left\|z_{n}-p\right\|^{2}+\left(1-\delta_{n}\right)\left\|S^{n} z_{n}-p\right\|^{2} \\
& -\delta_{n}\left(1-\delta_{n}\right)\left\|z_{n}-S^{n} z_{n}\right\|^{2}
\end{aligned}
$$

$$
\begin{aligned}
\leq & \delta_{n}\left\|z_{n}-p\right\|^{2}+\left(1-\delta_{n}\right) \\
& \times\left[\left(1+\gamma_{n}\right)\left\|z_{n}-p\right\|^{2}+\kappa\left\|z_{n}-S^{n} z_{n}\right\|^{2}+c_{n}\right] \\
& -\delta_{n}\left(1-\delta_{n}\right)\left\|z_{n}-S^{n} z_{n}\right\|^{2} \\
= & {\left[1+\gamma_{n}\left(1-\delta_{n}\right)\right]\left\|z_{n}-p\right\|^{2} } \\
& +\left(1-\delta_{n}\right)\left(\kappa-\delta_{n}\right)\left\|z_{n}-S^{n} z_{n}\right\|^{2}+\left(1-\delta_{n}\right) c_{n} \\
\leq & \left(1+\gamma_{n}\right)\left\|z_{n}-p\right\|^{2} \\
& +\left(1-\delta_{n}\right)\left(\kappa-\delta_{n}\right)\left\|z_{n}-S^{n} z_{n}\right\|^{2}+c_{n} \\
\leq & \left(1+\gamma_{n}\right)\left\|z_{n}-p\right\|^{2}+c_{n} .
\end{aligned}
$$

It follows from (42), (43), and the convexity of $\|\cdot\|^{2}$ that

$$
\begin{aligned}
\left\|y_{n}-p\right\|^{2}= & \|\left(1-\alpha_{n}-\beta_{n}\right)\left(x_{n}-p\right)+\alpha_{n}\left(k_{n}-p\right) \\
& +\beta_{n}\left(W_{n} z_{n}-p\right) \|^{2} \\
\leq & \left\|\left(1-\alpha_{n}-\beta_{n}\right)\right\| x_{n}-p\left\|^{2}+\alpha_{n}\right\| k_{n}-p \|^{2} \\
& +\beta_{n}\left\|W_{n} z_{n}-p\right\|^{2} \\
\leq & \left(1-\alpha_{n}-\beta_{n}\right)\left\|x_{n}-p\right\|^{2} \\
& +\alpha_{n}\left(\left(1+\gamma_{n}\right)\left\|z_{n}-p\right\|^{2}+c_{n}\right)+\beta_{n}\left\|z_{n}-p\right\|^{2} \\
\leq & \left(1-\alpha_{n}-\beta_{n}\right)\left\|x_{n}-p\right\|^{2} \\
& +\alpha_{n}\left(\left(1+\gamma_{n}\right)\left\|x_{n}-p\right\|^{2}+c_{n}\right)+\beta_{n}\left\|x_{n}-p\right\|^{2} \\
= & \left(1-\alpha_{n}\right)\left\|x_{n}-p\right\|^{2} \\
& +\alpha_{n}\left(\left(1+\gamma_{n}\right)\left\|x_{n}-p\right\|^{2}+c_{n}\right) \\
= & \left\|x_{n}-p\right\|^{2}+\alpha_{n} \gamma_{n}\left\|x_{n}-p\right\|^{2}+\alpha_{n} c_{n} \\
\leq & \left\|x_{n}-p\right\|^{2}+\gamma_{n}\left\|x_{n}-p\right\|^{2}+c_{n} \\
\leq & \left\|x_{n}-p\right\|^{2}+\theta_{n} .
\end{aligned}
$$

Hence, $p \in C_{n+1}$. This implies that $F \subset C_{n}$, for all $n \geq 1$.

Step 2. We prove that $\left\|x_{n}-k_{n}\right\| \rightarrow 0$ as $n \rightarrow \infty$.

Indeed, let $v=P_{F} x_{0}$. From $x_{n}=P_{C_{n}} x_{0}$ and $v \in F \subset C_{n}$, we obtain

$$
\left\|x_{n}-x_{0}\right\| \leq\left\|v-x_{0}\right\| .
$$

This implies that $\left\{x_{n}\right\}$ is bounded and, hence, $\left\{u_{n}\right\},\left\{z_{n}\right\},\left\{k_{n}\right\}$, and $\left\{y_{n}\right\}$ are also bounded. Since $x_{n+1} \in C_{n+1} \subset C_{n}$ and $x_{n}=$ $P_{C_{n}} x_{0}$, we have

$$
\left\|x_{n}-x_{0}\right\| \leq\left\|x_{n+1}-x_{0}\right\|, \quad \forall n \geq 1 .
$$

Therefore, $\lim _{n \rightarrow \infty}\left\|x_{n}-x_{0}\right\|$ exists. From $x_{n}=P_{C_{n}} x_{0}, x_{n+1} \epsilon$ $C_{n+1} \subset C_{n}$, by Proposition 5(ii) we obtain

$$
\left\|x_{n+1}-x_{n}\right\|^{2} \leq\left\|x_{0}-x_{n+1}\right\|^{2}-\left\|x_{0}-x_{n}\right\|^{2},
$$


which implies

$$
\lim _{n \rightarrow \infty}\left\|x_{n+1}-x_{n}\right\|=0 .
$$

It follows from $x_{n+1} \in C_{n+1}$ that $\left\|y_{n}-x_{n+1}\right\|^{2} \leq\left\|x_{n}-x_{n+1}\right\|^{2}+$ $\theta_{n}$ and hence

$$
\begin{aligned}
\left\|x_{n}-y_{n}\right\|^{2} & \leq 2\left(\left\|x_{n}-x_{n+1}\right\|^{2}+\left\|x_{n+1}-y_{n}\right\|^{2}\right) \\
& \leq 2\left(\left\|x_{n}-x_{n+1}\right\|^{2}+\left\|x_{n}-x_{n+1}\right\|^{2}+\theta_{n}\right) \\
& =2\left(2\left\|x_{n}-x_{n+1}\right\|^{2}+\theta_{n}\right) .
\end{aligned}
$$

From (48) and $\lim _{n \rightarrow \infty} \theta_{n}=0$, we have

$$
\lim _{n \rightarrow \infty}\left\|x_{n}-y_{n}\right\|=0 \text {. }
$$

Also, utilizing Lemma 7(b), we obtain from (36), (42), and (43) that

$$
\begin{aligned}
\left\|y_{n}-p\right\|^{2}= & \|\left(1-\alpha_{n}-\beta_{n}\right)\left(x_{n}-p\right)+\alpha_{n}\left(k_{n}-p\right) \\
& +\beta_{n}\left(W_{n} z_{n}-p\right) \|^{2} \\
\leq & \left(1-\alpha_{n}-\beta_{n}\right)\left\|x_{n}-p\right\|^{2}+\alpha_{n}\left\|k_{n}-p\right\|^{2} \\
& +\beta_{n}\left\|W_{n} z_{n}-p\right\|^{2} \\
& -\beta_{n}\left(1-\alpha_{n}-\beta_{n}\right)\left\|x_{n}-W_{n} z_{n}\right\|^{2} \\
\leq & \left(1-\alpha_{n}-\beta_{n}\right)\left\|x_{n}-p\right\|^{2} \\
& +\alpha_{n}\left[\left(1+\gamma_{n}\right)\left\|z_{n}-p\right\|^{2}+c_{n}\right]+\beta_{n}\left\|z_{n}-p\right\|^{2} \\
& -\beta_{n}\left(1-\alpha_{n}-\beta_{n}\right)\left\|x_{n}-W_{n} z_{n}\right\|^{2} \\
\leq & \left(1-\alpha_{n}-\beta_{n}\right)\left\|x_{n}-p\right\|^{2} \\
& +\alpha_{n}\left[\left(1+\gamma_{n}\right)\left\|x_{n}-p\right\|^{2}+c_{n}\right]+\beta_{n}\left\|x_{n}-p\right\|^{2} \\
& -\beta_{n}\left(1-\alpha_{n}-\beta_{n}\right)\left\|x_{n}-W_{n} z_{n}\right\|^{2} \\
= & \left(1-\alpha_{n}\right)\left\|x_{n}-p\right\|^{2} \\
& +\alpha_{n}\left[\left(1+\gamma_{n}\right)\left\|x_{n}-p\right\|^{2}+c_{n}\right] \\
& -\beta_{n}\left(1-\alpha_{n}-\beta_{n}\right)\left\|x_{n}-W_{n} z_{n}\right\|^{2} \\
= & \left\|x_{n}-p\right\|^{2}+\alpha_{n} \gamma_{n}\left\|x_{n}-p\right\|^{2}+\alpha_{n} c_{n} \\
& -\beta_{n}\left(1-\alpha_{n}-\beta_{n}\right)\left\|x_{n}-W_{n} z_{n}\right\|^{2} \\
\leq & \left\|x_{n}-p\right\|^{2}+\theta_{n}-\beta_{n}\left(1-\alpha_{n}-\beta_{n}\right)\left\|x_{n}-W_{n} z_{n}\right\|^{2}, \\
&
\end{aligned}
$$

which, hence, yields

$$
\begin{aligned}
& \beta_{n}\left(1-\alpha_{n}-\beta_{n}\right)\left\|x_{n}-W_{n} z_{n}\right\|^{2} \\
& \quad \leq\left\|x_{n}-p\right\|^{2}-\left\|y_{n}-p\right\|^{2}+\theta_{n} \\
& \quad \leq\left\|x_{n}-y_{n}\right\|\left(\left\|x_{n}-p\right\|+\left\|y_{n}-p\right\|\right)+\theta_{n} .
\end{aligned}
$$

Since $\theta_{n} \rightarrow 0$ and $\left\{x_{n}\right\},\left\{y_{n}\right\}$ are bounded, it follows from (50) and condition (i) that

$$
\lim _{n \rightarrow \infty}\left\|x_{n}-W_{n} z_{n}\right\|=0 .
$$

Note that

$$
y_{n}-x_{n}=\alpha_{n}\left(k_{n}-x_{n}\right)+\beta_{n}\left(W_{n} z_{n}-x_{n}\right),
$$

which leads to

$$
\begin{aligned}
\alpha_{n}\left\|k_{n}-x_{n}\right\| & =\left\|y_{n}-x_{n}-\beta_{n}\left(W_{n} z_{n}-x_{n}\right)\right\| \\
& \leq\left\|y_{n}-x_{n}\right\|+\beta_{n}\left\|W_{n} z_{n}-x_{n}\right\| \\
& \leq\left\|y_{n}-x_{n}\right\|+\left\|W_{n} z_{n}-x_{n}\right\| .
\end{aligned}
$$

So, from (50), (53), and $0<a \leq \alpha_{n} \leq 1$, we get

$$
\lim _{n \rightarrow \infty}\left\|x_{n}-k_{n}\right\|=0 .
$$

Step 3. We prove that $\left\|x_{n}-u_{n}\right\| \rightarrow 0,\left\|u_{n}-z_{n}\right\| \rightarrow 0, \| z_{n}-$ $W_{n} z_{n} \| \rightarrow 0$, and $\left\|z_{n}-S^{n} z_{n}\right\| \rightarrow 0$ as $n \rightarrow \infty$.

Indeed, from (42) and (43), it follows that

$$
\begin{aligned}
\left\|k_{n}-p\right\|^{2} \leq & {\left[1+\gamma_{n}\left(1-\delta_{n}\right)\right]\left\|z_{n}-p\right\|^{2} } \\
& +\left(1-\delta_{n}\right)\left(\kappa-\delta_{n}\right)\left\|z_{n}-S^{n} z_{n}\right\|^{2}+\left(1-\delta_{n}\right) c_{n} \\
\leq & \left\|z_{n}-p\right\|^{2}+\gamma_{n}\left\|z_{n}-p\right\|^{2}+c_{n} \\
\leq & \left\|z_{n}-p\right\|^{2}+\gamma_{n}\left\|x_{n}-p\right\|^{2}+c_{n} \\
\leq & \left\|z_{n}-p\right\|^{2}+\theta_{n} .
\end{aligned}
$$

Next, we prove that

$$
\lim _{n \rightarrow \infty}\left\|\Theta_{n}^{k} x_{n}-\Theta_{n}^{k-1} x_{n}\right\|=0, \quad k=1,2, \ldots, M .
$$

For $p \in F$, it follows from (18) that

$$
\begin{aligned}
\left\|\Theta_{n}^{k} x_{n}-p\right\|^{2}= & \left\|T_{r_{k, n}^{\left(F_{k}, \varphi_{k}\right)}\left(I-r_{k, n} A_{k}\right) \Theta_{n}^{k-1} x_{n}} \quad-T_{r_{k, n}}^{\left(F_{k}, \varphi_{k}\right)}\left(I-r_{k, n} A_{k}\right) p\right\|^{2} \\
\leq & \left\|\left(I-r_{k, n} A_{k}\right) \Theta_{n}^{k-1} x_{n}-\left(I-r_{k, n} A_{k}\right) p\right\|^{2} \\
\leq & \left\|\Theta_{n}^{k-1} x_{n}-p\right\|^{2} \\
& +r_{k, n}\left(r_{k, n}-2 \mu_{k}\right)\left\|A_{k} \Theta_{n}^{k-1} x_{n}-A_{k} p\right\|^{2} \\
\leq & \left\|x_{n}-p\right\|^{2} \\
& +r_{k, n}\left(r_{k, n}-2 \mu_{k}\right)\left\|A_{k} \Theta_{n}^{k-1} x_{n}-A_{k} p\right\|^{2} .
\end{aligned}
$$


By (40), (41), (57), and (59), we obtain

$$
\begin{aligned}
\left\|k_{n}-p\right\|^{2} & \leq\left\|z_{n}-p\right\|^{2}+\theta_{n} \\
& \leq\left\|u_{n}-p\right\|^{2}+\theta_{n} \\
& \leq\left\|\Theta_{n}^{k} x_{n}-p\right\|^{2}+\theta_{n} \\
& \leq\left\|x_{n}-p\right\|^{2}+r_{k, n}\left(r_{k, n}-2 \mu_{k}\right)\left\|A_{k} \Theta_{n}^{k-1} x_{n}-A_{k} p\right\|^{2} \\
& \quad+\theta_{n},
\end{aligned}
$$

which implies that

$$
\begin{aligned}
& r_{k, n}\left(2 \mu_{k}-r_{k, n}\right)\left\|A_{k} \Theta_{n}^{k-1} x_{n}-A_{k} p\right\|^{2} \\
& \quad \leq\left\|x_{n}-p\right\|^{2}-\left\|k_{n}-p\right\|^{2}+\theta_{n} \\
& \quad \leq\left\|x_{n}-k_{n}\right\|\left(\left\|x_{n}-p\right\|+\left\|k_{n}-p\right\|\right)+\theta_{n} .
\end{aligned}
$$

Since $\left\{r_{k, n}\right\} \subset\left[e_{k}, f_{k}\right] \subset\left(0,2 \mu_{k}\right), k \in\{1,2, \ldots, M\}$, $\lim _{n \rightarrow \infty} \theta_{n}=0$, and (56), we have

$$
\lim _{n \rightarrow \infty}\left\|A_{k} \Theta_{n}^{k-1} x_{n}-A_{k} p\right\|=0, \quad k=1,2, \ldots, M
$$

By Proposition 1(iii) and Lemma 7(a), we have

$$
\begin{aligned}
& \left\|\Theta_{n}^{k} x_{n}-p\right\|^{2} \\
& =\left\|T_{r_{k, n}}^{\left(F_{k}, \varphi_{k}\right)}\left(I-r_{k, n} A_{k}\right) \Theta_{n}^{k-1} x_{n}-T_{r_{k, n}}^{\left(F_{k}, \varphi_{k}\right)}\left(I-r_{k, n} A_{k}\right) p\right\|^{2} \\
& \leq\left\langle\left(I-r_{k, n} A_{k}\right) \Theta_{n}^{k-1} x_{n}-\left(I-r_{k, n} A_{k}\right) p, \Theta_{n}^{k} x_{n}-p\right\rangle \\
& =\frac{1}{2}\left(\left\|\left(I-r_{k, n} A_{k}\right) \Theta_{n}^{k-1} x_{n}-\left(I-r_{k, n} A_{k}\right) p\right\|^{2}\right. \\
& \quad+\left\|\Theta_{n}^{k} x_{n}-p\right\|^{2}-\|\left(I-r_{k, n} A_{k}\right) \Theta_{n}^{k-1} x_{n} \\
& \left.\quad-\left(I-r_{k, n} A_{k}\right) p-\left(\Theta_{n}^{k} x_{n}-p\right) \|^{2}\right) \\
& \leq \frac{1}{2}\left(\left\|\Theta_{n}^{k-1} x_{n}-p\right\|^{2}+\left\|\Theta_{n}^{k} x_{n}-p\right\|^{2}\right. \\
& \left.\quad-\left\|\Theta_{n}^{k-1} x_{n}-\Theta_{n}^{k} x_{n}-r_{k, n}\left(A_{k} \Theta_{n}^{k-1} x_{n}-A_{k} p\right)\right\|^{2}\right),
\end{aligned}
$$

which implies that

$$
\begin{aligned}
\left\|\Theta_{n}^{k} x_{n}-p\right\|^{2} & \\
\leq & \left\|\Theta_{n}^{k-1} x_{n}-p\right\|^{2} \\
& -\left\|\Theta_{n}^{k-1} x_{n}-\Theta_{n}^{k} x_{n}-r_{k, n}\left(A_{k} \Theta_{n}^{k-1} x_{n}-A_{k} p\right)\right\|^{2} \\
= & \left\|\Theta_{n}^{k-1} x_{n}-p\right\|^{2}-\left\|\Theta_{n}^{k-1} x_{n}-\Theta_{n}^{k} x_{n}\right\|^{2} \\
& -r_{k, n}^{2}\left\|A_{k} \Theta_{n}^{k-1} x_{n}-A_{k} p\right\|^{2} \\
& +2 r_{k, n}\left\langle\Theta_{n}^{k-1} x_{n}-\Theta_{n}^{k} x_{n}, A_{k} \Theta_{n}^{k-1} x_{n}-A_{k} p\right\rangle \\
\leq & \left\|\Theta_{n}^{k-1} x_{n}-p\right\|^{2}-\left\|\Theta_{n}^{k-1} x_{n}-\Theta_{n}^{k} x_{n}\right\|^{2} \\
& +2 r_{k, n}\left\|\Theta_{n}^{k-1} x_{n}-\Theta_{n}^{k} x_{n}\right\|\left\|A_{k} \Theta_{n}^{k-1} x_{n}-A_{k} p\right\| \\
\leq & \left\|x_{n}-p\right\|^{2}-\left\|\Theta_{n}^{k-1} x_{n}-\Theta_{n}^{k} x_{n}\right\|^{2} \\
& +2 r_{k, n}\left\|\Theta_{n}^{k-1} x_{n}-\Theta_{n}^{k} x_{n}\right\|\left\|A_{k} \Theta_{n}^{k-1} x_{n}-A_{k} p\right\| .
\end{aligned}
$$

Combining (57) and (64), we have

$$
\begin{aligned}
\| k_{n} & -p \|^{2} \\
\leq & \left\|z_{n}-p\right\|^{2}+\theta_{n} \\
\leq & \left\|u_{n}-p\right\|^{2}+\theta_{n} \\
\leq & \left\|\Theta_{n}^{k} x_{n}-p\right\|^{2}+\theta_{n} \\
\leq & \left\|x_{n}-p\right\|^{2}-\left\|\Theta_{n}^{k-1} x_{n}-\Theta_{n}^{k} x_{n}\right\|^{2} \\
& +2 r_{k, n}\left\|\Theta_{n}^{k-1} x_{n}-\Theta_{n}^{k} x_{n}\right\|\left\|A_{k} \Theta_{n}^{k-1} x_{n}-A_{k} p\right\|+\theta_{n},
\end{aligned}
$$

which implies

$$
\begin{aligned}
& \left\|\Theta_{n}^{k-1} x_{n}-\Theta_{n}^{k} x_{n}\right\|^{2} \\
& \leq\left\|x_{n}-p\right\|^{2}-\left\|k_{n}-p\right\|^{2} \\
& \quad+2 r_{k, n}\left\|\Theta_{n}^{k-1} x_{n}-\Theta_{n}^{k} x_{n}\right\|\left\|A_{k} \Theta_{n}^{k-1} x_{n}-A_{k} p\right\|+\theta_{n} \\
& \leq\left\|x_{n}-k_{n}\right\|\left(\left\|x_{n}-p\right\|+\left\|k_{n}-p\right\|\right) \\
& \quad+2 r_{k, n}\left\|\Theta_{n}^{k-1} x_{n}-\Theta_{n}^{k} x_{n}\right\|\left\|A_{k} \Theta_{n}^{k-1} x_{n}-A_{k} p\right\|+\theta_{n} .
\end{aligned}
$$

From $\lim _{n \rightarrow \infty} \theta_{n}=0,(56)$, and (62), we know that (58) holds. Hence, we obtain

$$
\begin{aligned}
\left\|x_{n}-u_{n}\right\|= & \left\|\Theta_{n}^{0} x_{n}-\Theta_{n}^{M} x_{n}\right\| \\
\leq & \left\|\Theta_{n}^{0} x_{n}-\Theta_{n}^{1} x_{n}\right\|+\left\|\Theta_{n}^{1} x_{n}-\Theta_{n}^{2} x_{n}\right\| \\
& +\cdots+\left\|\Theta_{n}^{M-1} x_{n}-\Theta_{n}^{M} x_{n}\right\|
\end{aligned}
$$


Abstract and Applied Analysis

9

Next, we show that $\lim _{n \rightarrow \infty}\left\|B_{i} \Omega_{n}^{i} u_{n}-B_{i} p\right\|=0, i=$ $1,2, \ldots, N$. It follows from Lemma 20 and (18) that

$$
\begin{aligned}
& \left\|\Omega_{n}^{i} u_{n}-p\right\|^{2} \\
& \quad=\left\|J_{R_{i}, \lambda_{i, n}}\left(I-\lambda_{i, n} B_{i}\right) \Omega_{n}^{i-1} u_{n}-J_{R_{i}, \lambda_{i, n}}\left(I-\lambda_{i, n} B_{i}\right) p\right\|^{2} \\
& \quad \leq\left\|\left(I-\lambda_{i, n} B_{i}\right) \Omega_{n}^{i-1} u_{n}-\left(I-\lambda_{i, n} B_{i}\right) p\right\|^{2} \\
& \quad \leq\left\|\Omega_{n}^{i-1} u_{n}-p\right\|^{2}+\lambda_{i, n}\left(\lambda_{i, n}-2 \eta_{i}\right)\left\|B_{i} \Omega_{n}^{i-1} u_{n}-B_{i} p\right\|^{2} \\
& \quad \leq\left\|u_{n}-p\right\|^{2}+\lambda_{i, n}\left(\lambda_{i, n}-2 \eta_{i}\right)\left\|B_{i} \Omega_{n}^{i-1} u_{n}-B_{i} p\right\|^{2} \\
& \quad \leq\left\|x_{n}-p\right\|^{2}+\lambda_{i, n}\left(\lambda_{i, n}-2 \eta_{i}\right)\left\|B_{i} \Omega_{n}^{i-1} u_{n}-B_{i} p\right\|^{2} .
\end{aligned}
$$

Combining (57) and (68), we have

$$
\begin{aligned}
\| k_{n} & -p \|^{2} \\
& \leq\left\|z_{n}-p\right\|^{2}+\theta_{n} \\
& \leq\left\|\Omega_{n}^{i} u_{n}-p\right\|^{2}+\theta_{n} \\
& \leq\left\|x_{n}-p\right\|^{2}+\lambda_{i, n}\left(\lambda_{i, n}-2 \eta_{i}\right)\left\|B_{i} \Omega_{n}^{i-1} u_{n}-B_{i} p\right\|^{2}+\theta_{n},
\end{aligned}
$$

which implies

$$
\begin{aligned}
& \lambda_{i, n}\left(2 \eta_{i}-\lambda_{i, n}\right)\left\|B_{i} \Omega_{n}^{i-1} u_{n}-B_{i} p\right\|^{2} \\
& \quad \leq\left\|x_{n}-p\right\|^{2}-\left\|k_{n}-p\right\|^{2}+\theta_{n} \\
& \quad \leq\left\|x_{n}-k_{n}\right\|\left(\left\|x_{n}-p\right\|+\left\|k_{n}-p\right\|\right)+\theta_{n} .
\end{aligned}
$$

From $\left\{\lambda_{i, n}\right\} \subset\left[a_{i}, b_{i}\right] \subset\left(0,2 \eta_{i}\right), i \in\{1,2, \ldots, N\}, \lim _{n \rightarrow \infty} \theta_{n}=$ 0 , and (56), we obtain

$$
\lim _{n \rightarrow \infty}\left\|B_{i} \Omega_{n}^{i-1} u_{n}-B_{i} p\right\|=0, \quad i=1,2, \ldots, N .
$$

By Lemmas 20 and 7(a), we obtain

$$
\begin{aligned}
& \left\|\Omega_{n}^{i} u_{n}-p\right\|^{2} \\
& =\left\|J_{R_{i}, \lambda_{i, n}}\left(I-\lambda_{i, n} B_{i}\right) \Omega_{n}^{i-1} u_{n}-J_{R_{i}, \lambda_{i, n}}\left(I-\lambda_{i, n} B_{i}\right) p\right\|^{2} \\
& \leq\left\langle\left(I-\lambda_{i, n} B_{i}\right) \Omega_{n}^{i-1} u_{n}-\left(I-\lambda_{i, n} B_{i}\right) p, \Omega_{n}^{i} u_{n}-p\right\rangle
\end{aligned}
$$

$$
\begin{aligned}
& =\frac{1}{2}\left(\left\|\left(I-\lambda_{i, n} B_{i}\right) \Omega_{n}^{i-1} u_{n}-\left(I-\lambda_{i, n} B_{i}\right) p\right\|^{2}+\left\|\Omega_{n}^{i} u_{n}-p\right\|^{2}\right. \\
& \left.-\left\|\left(I-\lambda_{i, n} B_{i}\right) \Omega_{n}^{i-1} u_{n}-\left(I-\lambda_{i, n} B_{i}\right) p-\left(\Omega_{n}^{i} u_{n}-p\right)\right\|^{2}\right) \\
& \leq \frac{1}{2}\left(\left\|\Omega_{n}^{i-1} u_{n}-p\right\|^{2}+\left\|\Omega_{n}^{i} u_{n}-p\right\|^{2}\right. \\
& \left.-\left\|\Omega_{n}^{i-1} u_{n}-\Omega_{n}^{i} u_{n}-\lambda_{i, n}\left(B_{i} \Omega_{n}^{i-1} u_{n}-B_{i} p\right)\right\|^{2}\right) \\
& \leq \frac{1}{2}\left(\left\|u_{n}-p\right\|^{2}+\left\|\Omega_{n}^{i} u_{n}-p\right\|^{2}\right. \\
& \left.-\left\|\Omega_{n}^{i-1} u_{n}-\Omega_{n}^{i} u_{n}-\lambda_{i, n}\left(B_{i} \Omega_{n}^{i-1} u_{n}-B_{i} p\right)\right\|^{2}\right) \\
& \leq \frac{1}{2}\left(\left\|x_{n}-p\right\|^{2}+\left\|\Omega_{n}^{i} u_{n}-p\right\|^{2}\right. \\
& \left.-\left\|\Omega_{n}^{i-1} u_{n}-\Omega_{n}^{i} u_{n}-\lambda_{i, n}\left(B_{i} \Omega_{n}^{i-1} u_{n}-B_{i} p\right)\right\|^{2}\right),
\end{aligned}
$$

which implies

$$
\begin{aligned}
\left\|\Omega_{n}^{i} u_{n}-p\right\|^{2} & \\
\leq & \left\|x_{n}-p\right\|^{2} \\
& -\left\|\Omega_{n}^{i-1} u_{n}-\Omega_{n}^{i} u_{n}-\lambda_{i, n}\left(B_{i} \Omega_{n}^{i-1} u_{n}-B_{i} p\right)\right\|^{2} \\
= & \left\|x_{n}-p\right\|^{2}-\left\|\Omega_{n}^{i-1} u_{n}-\Omega_{n}^{i} u_{n}\right\|^{2} \\
& \quad-\lambda_{i, n}^{2}\left\|B_{i} \Omega_{n}^{i-1} u_{n}-B_{i} p\right\|^{2} \\
& +2 \lambda_{i, n}\left\langle\Omega_{n}^{i-1} u_{n}-\Omega_{n}^{i} u_{n}, B_{i} \Omega_{n}^{i-1} u_{n}-B_{i} p\right\rangle \\
\leq & \left\|x_{n}-p\right\|^{2}-\left\|\Omega_{n}^{i-1} u_{n}-\Omega_{n}^{i} u_{n}\right\|^{2} \\
& +2 \lambda_{i, n}\left\|\Omega_{n}^{i-1} u_{n}-\Omega_{n}^{i} u_{n}\right\|\left\|B_{i} \Omega_{n}^{i-1} u_{n}-B_{i} p\right\| .
\end{aligned}
$$

Combining (57) and (73), we get

$$
\begin{aligned}
& \left\|k_{n}-p\right\|^{2} \\
& \leq\left\|z_{n}-p\right\|^{2}+\theta_{n} \\
& \leq\left\|\Omega_{n}^{i} u_{n}-p\right\|^{2}+\theta_{n} \\
& \leq\left\|x_{n}-p\right\|^{2}-\left\|\Omega_{n}^{i-1} u_{n}-\Omega_{n}^{i} u_{n}\right\|^{2} \\
& \quad+2 \lambda_{i, n}\left\|\Omega_{n}^{i-1} u_{n}-\Omega_{n}^{i} u_{n}\right\|\left\|B_{i} \Omega_{n}^{i-1} u_{n}-B_{i} p\right\|+\theta_{n},
\end{aligned}
$$

which implies

$$
\begin{aligned}
& \left\|\Omega_{n}^{i-1} u_{n}-\Omega_{n}^{i} u_{n}\right\|^{2} \\
& \quad \leq\left\|x_{n}-p\right\|^{2}-\left\|k_{n}-p\right\|^{2} \\
& \quad+2 \lambda_{i, n}\left\|\Omega_{n}^{i-1} u_{n}-\Omega_{n}^{i} u_{n}\right\|\left\|B_{i} \Omega_{n}^{i-1} u_{n}-B_{i} p\right\|+\theta_{n}
\end{aligned}
$$




$$
\begin{aligned}
\leq & \left\|x_{n}-k_{n}\right\|\left(\left\|x_{n}-p\right\|+\left\|k_{n}-p\right\|\right) \\
& +2 \lambda_{i, n}\left\|\Omega_{n}^{i-1} u_{n}-\Omega_{n}^{i} u_{n}\right\|\left\|B_{i} \Omega_{n}^{i-1} u_{n}-B_{i} p\right\|+\theta_{n} .
\end{aligned}
$$

From (56), (71), and $\lim _{n \rightarrow \infty} \theta_{n}=0$, we have

$$
\lim _{n \rightarrow \infty}\left\|\Omega_{n}^{i-1} u_{n}-\Omega_{n}^{i} u_{n}\right\|=0, \quad i=1,2, \ldots, N .
$$

From (76), we get

$$
\begin{aligned}
\left\|u_{n}-z_{n}\right\|= & \left\|\Omega_{n}^{0} u_{n}-\Omega_{n}^{N} u_{n}\right\| \\
\leq & \left\|\Omega_{n}^{0} u_{n}-\Omega_{n}^{1} u_{n}\right\|+\left\|\Omega_{n}^{1} u_{n}-\Omega_{n}^{2} u_{n}\right\| \\
& +\cdots+\left\|\Omega_{n}^{N-1} u_{n}-\Omega_{n}^{N} u_{n}\right\| \\
& \longrightarrow 0 \text { as } n \longrightarrow \infty .
\end{aligned}
$$

By (67) and (77), we have

$$
\begin{aligned}
\left\|x_{n}-z_{n}\right\| & \leq\left\|x_{n}-u_{n}\right\|+\left\|u_{n}-z_{n}\right\| \\
& \longrightarrow 0 \text { as } n \longrightarrow \infty .
\end{aligned}
$$

From (48) and (78), we have

$$
\begin{aligned}
\left\|z_{n+1}-z_{n}\right\| \leq & \left\|z_{n+1}-x_{n+1}\right\|+\left\|x_{n+1}-x_{n}\right\| \\
& +\left\|x_{n}-z_{n}\right\| \longrightarrow 0 \text { as } n \longrightarrow \infty .
\end{aligned}
$$

By (56), (67), and (77), we get

$$
\begin{aligned}
\left\|k_{n}-z_{n}\right\| & \leq\left\|k_{n}-x_{n}\right\|+\left\|x_{n}-u_{n}\right\|+\left\|u_{n}-z_{n}\right\| \\
& \longrightarrow 0 \quad \text { as } n \longrightarrow \infty .
\end{aligned}
$$

We observe that

$$
k_{n}-z_{n}=\left(1-\delta_{n}\right)\left(S^{n} z_{n}-z_{n}\right) .
$$

From $\delta_{n} \leq d<1$ and (80), we have

$$
\lim _{n \rightarrow \infty}\left\|S^{n} z_{n}-z_{n}\right\|=0 .
$$

We note that

$$
\begin{aligned}
\left\|S^{n} z_{n}-S^{n+1} z_{n}\right\| \leq & \left\|S^{n} z_{n}-z_{n}\right\|+\left\|z_{n}-z_{n+1}\right\| \\
& +\left\|z_{n+1}-S^{n+1} z_{n+1}\right\| \\
& +\left\|S^{n+1} z_{n+1}-S^{n+1} z_{n}\right\| .
\end{aligned}
$$

From (79), (82), and Lemma 9, we obtain

$$
\lim _{n \rightarrow \infty}\left\|S^{n} z_{n}-S^{n+1} z_{n}\right\|=0 .
$$

On the other hand, we note that

$$
\begin{aligned}
\left\|z_{n}-S z_{n}\right\| \leq & \left\|z_{n}-S^{n} z_{n}\right\|+\left\|S^{n} z_{n}-S^{n+1} z_{n}\right\| \\
& +\left\|S^{n+1} z_{n}-S z_{n}\right\| .
\end{aligned}
$$

From (82), (84), and the uniform continuity of $S$, we have

$$
\lim _{n \rightarrow \infty}\left\|z_{n}-S z_{n}\right\|=0 .
$$

In addition, note that

$$
\begin{aligned}
\left\|z_{n}-W z_{n}\right\| & \leq\left\|z_{n}-W_{n} z_{n}\right\|+\left\|W_{n} z_{n}-W z_{n}\right\| \\
& \leq\left\|z_{n}-x_{n}\right\|+\left\|x_{n}-W_{n} z_{n}\right\|+\left\|W_{n} z_{n}-W z_{n}\right\| .
\end{aligned}
$$

So, from (53), (78), and [4, Remark 3.2], it follows that

$$
\lim _{n \rightarrow \infty}\left\|z_{n}-W z_{n}\right\|=0
$$

Step 4. Finally we prove that $x_{n} \rightarrow v=P_{F} x_{0}$ as $n \rightarrow \infty$.

Indeed, since $\left\{x_{n}\right\}$ is bounded, there exists a subsequence $\left\{x_{n_{i}}\right\}$ which converges weakly to some $w$. From (58) and (76)(78), we have that $\Theta_{n_{i}}^{k} x_{n_{i}} \rightarrow w, \Omega_{n_{i}}^{m} u_{n_{i}} \rightarrow w$ and $z_{n_{i}} \rightarrow w$, where $k \in\{1,2, \ldots, M\}$ and $m \in\{1,2, \ldots, N\}$. Since $S$ is uniformly continuous, by (86), we get $\lim _{n \rightarrow \infty}\left\|z_{n}-S^{m} z_{n}\right\|=$ 0 , for any $m \geq 1$. Hence, from Lemma 11, we obtain $w \in$ $\operatorname{Fix}(S)$. In the meantime, utilizing Lemma 19, we deduce from (88) and $z_{n_{i}} \rightarrow w$ that $w \in \operatorname{Fix}(W)=\bigcap_{n=1}^{\infty} \operatorname{Fix}\left(T_{n}\right)$. Next, we prove that $w \in \bigcap_{m=1}^{N} I\left(B_{m}, R_{m}\right)$. As a matter of fact, since $B_{m}$ is $\eta_{m}$-inverse strongly monotone, $B_{m}$ is a monotone and Lipschitz continuous mapping. It follows from Lemma 23 that $R_{m}+B_{m}$ is maximal monotone. Let $(v, g) \in G\left(R_{m}+B_{m}\right)$; that is, $g-B_{m} v \in R_{m} v$. Again, since $\Omega_{n}^{m} u_{n}=J_{R_{m}, \lambda_{m, n}}(I-$ $\left.\lambda_{m, n} B_{m}\right) \Lambda_{n}^{m-1} u_{n}, n \geq 1, m \in\{1,2, \ldots, N\}$, we have

$$
\Omega_{n}^{m-1} u_{n}-\lambda_{m, n} B_{m} \Omega_{n}^{m-1} u_{n} \in\left(I+\lambda_{m, n} R_{m}\right) \Omega_{n}^{m} u_{n}
$$

that is,

$$
\frac{1}{\lambda_{m, n}}\left(\Omega_{n}^{m-1} u_{n}-\Omega_{n}^{m} u_{n}-\lambda_{m, n} B_{m} \Omega_{n}^{m-1} u_{n}\right) \in R_{m} \Omega_{n}^{m} u_{n} .
$$

In terms of the monotonicity of $R_{m}$, we get

$$
\begin{aligned}
& \left\langle v-\Omega_{n}^{m} u_{n}, g-B_{m} v\right. \\
& \left.\quad-\frac{1}{\lambda_{m, n}}\left(\Omega_{n}^{m-1} u_{n}-\Omega_{n}^{m} u_{n}-\lambda_{m, n} B_{m} \Omega_{n}^{m-1} u_{n}\right)\right\rangle \geq 0
\end{aligned}
$$

and, hence,

$$
\begin{aligned}
& \left\langle v-\Omega_{n}^{m} u_{n}, g\right\rangle \\
& \geq\left\langle v-\Omega_{n}^{m} u_{n}, B_{m} v\right. \\
& \left.\quad+\frac{1}{\lambda_{m, n}}\left(\Omega_{n}^{m-1} u_{n}-\Omega_{n}^{m} u_{n}-\lambda_{m, n} B_{m} \Omega_{n}^{m-1} u_{n}\right)\right\rangle
\end{aligned}
$$




$$
\begin{aligned}
= & \left\langle v-\Omega_{n}^{m} u_{n}, B_{m} v-B_{m} \Omega_{n}^{m} u_{n}+B_{m} \Omega_{n}^{m} u_{n}-B_{m} \Omega_{n}^{m-1} u_{n}\right. \\
& \left.+\frac{1}{\lambda_{m, n}}\left(\Omega_{n}^{m-1} u_{n}-\Omega_{n}^{m} u_{n}\right)\right\rangle \\
\geq & \left\langle v-\Omega_{n}^{m} u_{n}, B_{m} \Omega_{n}^{m} u_{n}-B_{m} \Omega_{n}^{m-1} u_{n}\right\rangle \\
& +\left\langle v-\Omega_{n}^{m} u_{n}, \frac{1}{\lambda_{m, n}}\left(\Omega_{n}^{m-1} u_{n}-\Omega_{n}^{m} u_{n}\right)\right\rangle .
\end{aligned}
$$

In particular,

$$
\begin{aligned}
\langle v- & \left.\Omega_{n_{i}}^{m} u_{n_{i}}, g\right\rangle \\
\geq & \left\langle v-\Omega_{n_{i}}^{m} u_{n_{i}}, B_{m} \Omega_{n_{i}}^{m} u_{n_{i}}-B_{m} \Omega_{n_{i}}^{m-1} u_{n_{i}}\right\rangle \\
& \quad\left\langle v-\Omega_{n_{i}}^{m} u_{n_{i}}, \frac{1}{\lambda_{m, n_{i}}}\left(\Omega_{n_{i}}^{m-1} u_{n_{i}}-\Omega_{n_{i}}^{m} u_{n_{i}}\right)\right\rangle .
\end{aligned}
$$

Since $\left\|\Omega_{n}^{m} u_{n}-\Omega_{n}^{m-1} u_{n}\right\| \rightarrow 0$ (due to (76)) and $\| B_{m} \Omega_{n}^{m} u_{n}-$ $B_{m} \Omega_{n}^{m-1} u_{n} \| \rightarrow 0$ (due to the Lipschitz continuity of $B_{m}$ ), we conclude from $\Omega_{n_{i}}^{m} u_{n_{i}} \rightarrow w$ and condition (ii) that

$$
\lim _{i \rightarrow \infty}\left\langle v-\Omega_{n_{i}}^{m} u_{n_{i}}, g\right\rangle=\langle v-w, g\rangle \geq 0 .
$$

It follows from the maximal monotonicity of $B_{m}+R_{m}$ that $0 \in\left(R_{m}+B_{m}\right) w$; that is, $w \in I\left(B_{m}, R_{m}\right)$. Therefore, $w \in \bigcap_{m=1}^{N} I\left(B_{m}, R_{m}\right)$. Next, we prove that $w \in \bigcap_{k=1}^{M} \operatorname{GMEP}\left(F_{k}, \varphi_{k}, A_{k}\right)$. Since $\Theta_{n}^{k} x_{n}=T_{r_{k, n}}^{\left(F_{k}, \varphi_{k}\right)}(I-$ $\left.r_{k, n} A_{k}\right) \Theta_{n}^{k-1} x_{n}, n \geq 1, k \in\{1,2, \ldots, M\}$, we have

$$
\begin{aligned}
& F_{k}\left(\Theta_{n}^{k} x_{n}, y\right)+\varphi_{k}(y)-\varphi_{k}\left(\Theta_{n}^{k} x_{n}\right)+\left\langle A_{k} \Theta_{n}^{k-1} x_{n}, y-\Theta_{n}^{k} x_{n}\right\rangle \\
& \quad+\frac{1}{r_{k, n}}\left\langle y-\Theta_{n}^{k} x_{n}, \Theta_{n}^{k} x_{n}-\Theta_{n}^{k-1} x_{n}\right\rangle \geq 0 .
\end{aligned}
$$

By (A2), we have

$$
\begin{aligned}
& \varphi_{k}(y)-\varphi_{k}\left(\Theta_{n}^{k} x_{n}\right)+\left\langle A_{k} \Theta_{n}^{k-1} x_{n}, y-\Theta_{n}^{k} x_{n}\right\rangle \\
& +\frac{1}{r_{k, n}}\left\langle y-\Theta_{n}^{k} x_{n}, \Theta_{n}^{k} x_{n}-\Theta_{n}^{k-1} x_{n}\right\rangle \geq F_{k}\left(y, \Theta_{n}^{k} x_{n}\right) .
\end{aligned}
$$

Let $z_{t}=t y+(1-t) w$, for all $t \in(0,1]$ and $y \in C$. This implies that $z_{t} \in C$. Then, we have

$$
\begin{aligned}
\left\langle z_{t}\right. & \left.-\Theta_{n}^{k} x_{n}, A_{k} z_{t}\right\rangle \\
\geq & \varphi_{k}\left(\Theta_{n}^{k} x_{n}\right)-\varphi_{k}\left(z_{t}\right)+\left\langle z_{t}-\Theta_{n}^{k} x_{n}, A_{k} z_{t}\right\rangle \\
& -\left\langle z_{t}-\Theta_{n}^{k} x_{n}, A_{k} \Theta_{n}^{k-1} x_{n}\right\rangle \\
& -\left\langle z_{t}-\Theta_{n}^{k} x_{n}, \frac{\Theta_{n}^{k} x_{n}-\Theta_{n}^{k-1} x_{n}}{r_{k, n}}\right\rangle+F_{k}\left(z_{t}, \Theta_{n}^{k} x_{n}\right)
\end{aligned}
$$

$$
\begin{aligned}
= & \varphi_{k}\left(\Theta_{n}^{k} x_{n}\right)-\varphi_{k}\left(z_{t}\right)+\left\langle z_{t}-\Theta_{n}^{k} x_{n}, A_{k} z_{t}-A_{k} \Theta_{n}^{k} x_{n}\right\rangle \\
& +\left\langle z_{t}-\Theta_{n}^{k} x_{n}, A_{k} \Theta_{n}^{k} x_{n}-A_{k} \Theta_{n}^{k-1} x_{n}\right\rangle \\
& -\left\langle z_{t}-\Theta_{n}^{k} x_{n}, \frac{\Theta_{n}^{k} x_{n}-\Theta_{n}^{k-1} x_{n}}{r_{k, n}}\right\rangle+F_{k}\left(z_{t}, \Theta_{n}^{k} x_{n}\right) .
\end{aligned}
$$

By (58), we have $\left\|A_{k} \Theta_{n}^{k} x_{n}-A_{k} \Theta_{n}^{k-1} x_{n}\right\| \rightarrow 0$ as $n \rightarrow \infty$. Furthermore, by the monotonicity of $A_{k}$, we obtain $\left\langle z_{t}-\right.$ $\left.\Theta_{n}^{k} x_{n}, A_{k} z_{t}-A_{k} \Theta_{n}^{k} x_{n}\right\rangle \geq 0$. Then, by (A4), we obtain

$$
\left\langle z_{t}-w, A_{k} z_{t}\right\rangle \geq \varphi_{k}(w)-\varphi_{k}\left(z_{t}\right)+F_{k}\left(z_{t}, w\right) .
$$

Utilizing (A1), (A4), and (98), we obtain

$$
\begin{aligned}
0= & F_{k}\left(z_{t}, z_{t}\right)+\varphi_{k}\left(z_{t}\right)-\varphi_{k}\left(z_{t}\right) \\
\leq & t F_{k}\left(z_{t}, y\right)+(1-t) F_{k}\left(z_{t}, w\right)+t \varphi_{k}(y) \\
& +(1-t) \varphi_{k}(w)-\varphi_{k}\left(z_{t}\right) \\
\leq & t\left[F_{k}\left(z_{t}, y\right)+\varphi_{k}(y)-\varphi_{k}\left(z_{t}\right)\right]+(1-t)\left\langle z_{t}-w, A_{k} z_{t}\right\rangle \\
= & t\left[F_{k}\left(z_{t}, y\right)+\varphi_{k}(y)-\varphi_{k}\left(z_{t}\right)\right]+(1-t) t\left\langle y-w, A_{k} z_{t}\right\rangle,
\end{aligned}
$$

and, hence,

$$
0 \leq F_{k}\left(z_{t}, y\right)+\varphi_{k}(y)-\varphi_{k}\left(z_{t}\right)+(1-t)\left\langle y-w, A_{k} z_{t}\right\rangle .
$$

Letting $t \rightarrow 0$, we have, for each $y \in C$,

$$
0 \leq F_{k}(w, y)+\varphi_{k}(y)-\varphi_{k}(w)+\left\langle y-w, A_{k} w\right\rangle .
$$

This implies that $w \in \operatorname{GMEP}\left(F_{k}, \varphi_{k}, A_{k}\right)$ and, hence, $w \in \bigcap_{k=1}^{M} \operatorname{GMEP}\left(F_{k}, \varphi_{k}, A_{k}\right)$. Consequently, $w \in F=$ $\bigcap_{n=1}^{\infty} \operatorname{Fix}\left(T_{n}\right) \cap \bigcap_{k=1}^{M} \operatorname{GMEP}\left(F_{k}, \varphi_{k}, A_{k}\right) \cap \bigcap_{m=1}^{N} I\left(B_{m}, R_{m}\right) \cap$ $\operatorname{Fix}(S)$. This shows that $\omega_{w}\left(\left\{x_{n}\right\}\right) \subset F$. From (45) and Lemma 16, we infer that $x_{n} \rightarrow v=P_{F} x_{0}$ as $n \rightarrow \infty$. This completes the proof.

\section{Weak Convergence Theorem}

In this section, we prove a weak convergence theorem for an iterative algorithm for finding a common element of the set of solutions of the set of solutions of finite generalized mixed equilibrium problems, the set of solutions of finite variational inclusions for maximal monotone and inverse strong monotone mappings, and the set of common fixed points of infinite nonexpansive mappings and asymptotically $\kappa$-strict pseudocontractive mapping $S: C \rightarrow C$ in the intermediate sense in a real Hilbert space. This iterative algorithm is based on the extragradient method and Manntype iterative method.

Theorem 25. Let $C$ be a nonempty closed convex subset of a real Hilbert space $H$. Let $M, N$ be two integers. Let $F_{k}$ be a bifunction from $C \times C$ to $\mathbf{R}$ satisfying (A1)-(A4) and let 
$\varphi_{k}: C \rightarrow \mathbf{R} \cup\{+\infty\}$ be a proper lower semicontinuous and convex function, where $k \in\{1,2, \ldots, M\}$. Let $R_{i}: C \rightarrow$ $2^{H}$ be a maximal monotone mapping and let $A_{k}: H \rightarrow$ $H$ and $B_{i}: C \rightarrow H$ be $\mu_{k}$-inverse strongly monotone and $\eta_{i}$-inverse strongly monotone, respectively, where $k \in$ $\{1,2, \ldots, M\}, i \in\{1,2, \ldots, N\}$. Let $S: C \rightarrow C$ be a uniformly continuous asymptotically $\kappa$-strict pseudocontractive mapping in the intermediate sense, for some $0 \leq \kappa<1$, with sequences $\left\{\gamma_{n}\right\} \subset[0, \infty)$ and $\left\{c_{n}\right\} \subset[0, \infty)$. Let $\left\{T_{n}\right\}_{n=1}^{\infty}$ be a sequence of nonexpansive self-mappings on $C$ and $\left\{\lambda_{n}\right\}$ be a sequence in $(0, b]$, for some $b \in(0,1)$. Assume that $F:=\bigcap_{n=1}^{\infty} \operatorname{Fix}\left(T_{n}\right) \cap$ $\bigcap_{k=1}^{M} \operatorname{GMEP}\left(F_{k}, \varphi_{k}, A_{k}\right) \cap \bigcap_{i=1}^{N} I\left(B_{i}, R_{i}\right) \cap \operatorname{Fix}(S)$ is nonempty and that either (B1) or (B2) holds. Let $\left\{\alpha_{n}\right\},\left\{\beta_{n}\right\}$, and $\left\{\delta_{n}\right\}$ be sequences in $[0,1]$ such that $\alpha_{n}+\beta_{n} \leq 1,0<a \leq \alpha_{n} \leq 1$ and $\kappa \leq \delta_{n} \leq d<1$. Pick any $x_{1} \in H$ and let $\left\{x_{n}\right\}$ be a sequence generated by the following algorithm:

$$
\begin{aligned}
u_{n}= & T_{r_{M, n}}^{\left(F_{M}, \varphi_{M}\right)}\left(I-r_{M, n} A_{M}\right) T_{r_{M-1, n}}^{\left(F_{M-1}, \varphi_{M-1}\right)} \\
& \times\left(I-r_{M-1, n} A_{M-1}\right) \cdots T_{\left.r_{1, n}, \varphi_{1}\right)}^{\left(F_{1}\right)}\left(I-r_{1, n} A_{1}\right) x_{n} \\
z_{n}= & J_{R_{N}, \lambda_{N, n}}\left(I-\lambda_{N, n} B_{N}\right) J_{R_{N-1}, \lambda_{N-1, n}} \\
& \times\left(I-\lambda_{N-1, n} B_{N-1}\right) \cdots J_{R_{1}, \lambda_{1, n}}\left(I-\lambda_{1, n} B_{1}\right) u_{n} \\
k_{n}= & \delta_{n} z_{n}+\left(1-\delta_{n}\right) S^{n} z_{n}, \\
y_{n}= & \left(1-\alpha_{n}-\beta_{n}\right) x_{n}+\alpha_{n} k_{n}+\beta_{n} W_{n} z_{n}
\end{aligned}
$$

where $W_{n}$ is the $W$-mapping generated by (2.2). Assume that the following conditions hold:

(i) $\sum_{n=1}^{\infty} \gamma_{n}<\infty$ and $\sum_{n=1}^{\infty} c_{n}<\infty$;

(ii) $0<\liminf _{n \rightarrow \infty} \beta_{n} \leq \limsup _{n \rightarrow \infty}\left(\alpha_{n}+\beta_{n}\right)<1$;

(iii) $\left\{\lambda_{i, n}\right\} \subset\left[a_{i}, b_{i}\right] \subset\left(0,2 \eta_{i}\right), \forall i \in\{1,2, \ldots, N\}$;

(iv) $\left\{r_{k, n}\right\} \subset\left[e_{k}, f_{k}\right] \subset\left(0,2 \mu_{k}\right), \forall k \in\{1,2, \ldots, M\}$.

Then, $\left\{x_{n}\right\}$ converges weakly to $w=\lim _{n \rightarrow \infty} P_{F} x_{n}$.

Proof. First, let us show that $\lim _{n \rightarrow \infty}\left\|x_{n}-p\right\|$ exists, for any $p \in F$. Put

$$
\begin{aligned}
\Theta_{n}^{k}= & T_{r_{k, n}}^{\left(F_{k}, \varphi_{k}\right)}\left(I-r_{k, n} A_{k}\right) T_{r_{k-1, n}}^{\left(F_{k-1}, \varphi_{k-1}\right)} \\
& \times\left(I-r_{k-1, n} A_{k-1}\right) \cdots T_{r_{1, n}}^{\left(F_{1}, \varphi_{1}\right)}\left(I-r_{1, n} A_{1}\right) x_{n},
\end{aligned}
$$

for all $k \in\{1,2, \ldots, M\}, n \geq 1$;

$$
\begin{aligned}
\Omega_{n}^{i}= & J_{R_{i}, \lambda_{i, n}}\left(I-\lambda_{i, n} B_{i}\right) J_{R_{i-1}, \lambda_{i-1, n}} \\
& \times\left(I-\lambda_{i-1, n} B_{i-1}\right) \cdots J_{R_{1}, \lambda_{1, n}}\left(I-\lambda_{1, n} B_{1}\right),
\end{aligned}
$$

for all $i \in\{1,2, \ldots, N\}, n \geq 1, \Theta_{n}^{0}=\Omega_{n}^{0}=I$, where $I$ is the identity mapping on $H$. Then, we have that $u_{n}=\Theta_{n}^{M} x_{n}$ and
$z_{n}=\Omega_{n}^{N} u_{n}$. Take $p \in F$ arbitrarily. Similar to the proof of Theorem 24, we obtain that

$$
\begin{aligned}
& \left\|u_{n}-p\right\| \leq\left\|x_{n}-p\right\|, \\
& \left\|z_{n}-p\right\| \leq\left\|u_{n}-p\right\|, \\
& \left\|\Theta_{n}^{k} x_{n}-p\right\|^{2} \leq\left\|x_{n}-p\right\|^{2} \\
& +r_{k, n}\left(r_{k, n}-2 \mu_{k}\right)\left\|A_{k} \Theta_{n}^{k-1} x_{n}-A_{k} p\right\|^{2}, \\
& k \in\{1,2, \ldots, M\} \text {, } \\
& \left\|\Theta_{n}^{k} x_{n}-p\right\|^{2} \leq\left\|x_{n}-p\right\|^{2}-\left\|\Theta_{n}^{k-1} x_{n}-\Theta_{n}^{k} x_{n}\right\|^{2} \\
& +2 r_{k, n}\left\|\Theta_{n}^{k-1} x_{n}-\Theta_{n}^{k} x_{n}\right\| \\
& \times\left\|A_{k} \Theta_{n}^{k-1} x_{n}-A_{k} p\right\|, \quad k=1,2, \ldots, M, \\
& +\lambda_{i, n}\left(\lambda_{i, n}-2 \eta_{i}\right)\left\|B_{i} \Omega_{n}^{i-1} u_{n}-B_{i} p\right\|^{2}, \\
& i \in\{1,2, \ldots, N\} \text {, } \\
& \left\|\Omega_{n}^{i} u_{n}-p\right\|^{2} \leq\left\|x_{n}-p\right\|^{2}-\left\|\Omega_{n}^{i-1} u_{n}-\Omega_{n}^{i} u_{n}\right\|^{2} \\
& +2 \lambda_{i, n}\left\|\Omega_{n}^{i-1} u_{n}-\Omega_{n}^{i} u_{n}\right\| \\
& \times\left\|B_{i} \Omega_{n}^{i-1} u_{n}-B_{i} p\right\|, \quad i \in\{1,2, \ldots, N\} .
\end{aligned}
$$

We observe that

$$
\begin{aligned}
\left\|k_{n}-p\right\|^{2}= & \left\|\delta_{n}\left(z_{n}-p\right)+\left(1-\delta_{n}\right)\left(S^{n} z_{n}-p\right)\right\|^{2} \\
= & \delta_{n}\left\|z_{n}-p\right\|^{2}+\left(1-\delta_{n}\right)\left\|S^{n} z_{n}-p\right\|^{2} \\
& -\delta_{n}\left(1-\delta_{n}\right)\left\|z_{n}-S^{n} z_{n}\right\|^{2} \\
\leq & \delta_{n}\left\|z_{n}-p\right\|^{2}+\left(1-\delta_{n}\right) \\
& \times\left[\left(1+\gamma_{n}\right)\left\|z_{n}-p\right\|^{2}+\kappa\left\|z_{n}-S^{n} z_{n}\right\|^{2}+c_{n}\right] \\
& -\delta_{n}\left(1-\delta_{n}\right)\left\|z_{n}-S^{n} z_{n}\right\|^{2} \\
= & {\left[1+\gamma_{n}\left(1-\delta_{n}\right)\right]\left\|z_{n}-p\right\|^{2} } \\
& +\left(1-\delta_{n}\right)\left(\kappa-\delta_{n}\right)\left\|z_{n}-S^{n} z_{n}\right\|^{2}+\left(1-\delta_{n}\right) c_{n} \\
\leq & \left(1+\gamma_{n}\right)\left\|z_{n}-p\right\|^{2}+c_{n} .
\end{aligned}
$$

It follows from (105), (106), and (111) that

$$
\begin{aligned}
& \left\|x_{n+1}-p\right\|^{2} \\
& =\left\|\left(1-\alpha_{n}-\beta_{n}\right)\left(x_{n}-p\right)+\alpha_{n}\left(k_{n}-p\right)+\beta_{n}\left(W_{n} z_{n}-p\right)\right\|^{2}
\end{aligned}
$$


Abstract and Applied Analysis

13

$$
\begin{aligned}
\leq & \left(1-\alpha_{n}-\beta_{n}\right)\left\|x_{n}-p\right\|^{2}+\alpha_{n}\left\|k_{n}-p\right\|^{2} \\
& +\beta_{n}\left\|W_{n} z_{n}-p\right\|^{2} \\
\leq & \left(1-\alpha_{n}-\beta_{n}\right)\left\|x_{n}-p\right\|^{2}+\alpha_{n}\left[\left(1+\gamma_{n}\right)\left\|z_{n}-p\right\|^{2}+c_{n}\right] \\
& +\beta_{n}\left\|z_{n}-p\right\|^{2} \\
\leq & \left(1-\alpha_{n}-\beta_{n}\right)\left\|x_{n}-p\right\|^{2}+\alpha_{n}\left[\left(1+\gamma_{n}\right)\left\|x_{n}-p\right\|^{2}+c_{n}\right] \\
& +\beta_{n}\left\|x_{n}-p\right\|^{2} \\
= & \left(1-\alpha_{n}\right)\left\|x_{n}-p\right\|^{2}+\alpha_{n}\left[\left(1+\gamma_{n}\right)\left\|x_{n}-p\right\|^{2}+c_{n}\right] \\
= & \left(1+\alpha_{n} \gamma_{n}\right)\left\|x_{n}-p\right\|^{2}+\alpha_{n} c_{n} \\
\leq & \left(1+\gamma_{n}\right)\left\|x_{n}-p\right\|^{2}+c_{n} .
\end{aligned}
$$

By Lemma 13 and condition (i), we deduce that $\lim _{n \rightarrow \infty} \| x_{n}-$ $p \|$ exists. Hence, $\left\{x_{n}\right\}$ is bounded and so are $\left\{u_{n}\right\},\left\{z_{n}\right\}$, and $\left\{k_{n}\right\}$.

In addition, by Lemma 7(b), we obtain from (105), (106), and (111) that

$$
\begin{aligned}
& \left\|x_{n+1}-p\right\|^{2} \\
& =\left\|\left(1-\alpha_{n}-\beta_{n}\right)\left(x_{n}-p\right)+\alpha_{n}\left(k_{n}-p\right)+\beta_{n}\left(W_{n} z_{n}-p\right)\right\|^{2} \\
& =\left(1-\alpha_{n}-\beta_{n}\right)\left\|x_{n}-p\right\|^{2} \\
& +\left(\alpha_{n}+\beta_{n}\right)\left\|\frac{\alpha_{n}\left(k_{n}-p\right)+\beta_{n}\left(W_{n} z_{n}-p\right)}{\alpha_{n}+\beta_{n}}\right\|^{2} \\
& -\left(\alpha_{n}+\beta_{n}\right)\left(1-\alpha_{n}-\beta_{n}\right)\left\|\frac{\alpha_{n}\left(k_{n}-x_{n}\right)+\beta_{n}\left(W_{n} z_{n}-x_{n}\right)}{\alpha_{n}+\beta_{n}}\right\|^{2} \\
& \leq\left(1-\alpha_{n}-\beta_{n}\right)\left\|x_{n}-p\right\|^{2} \\
& +\left(\alpha_{n}+\beta_{n}\right)\left[\frac{\alpha_{n}}{\alpha_{n}+\beta_{n}}\left\|k_{n}-p\right\|^{2}+\frac{\beta_{n}}{\alpha_{n}+\beta_{n}}\left\|W_{n} z_{n}-p\right\|^{2}\right] \\
& -\left(\alpha_{n}+\beta_{n}\right)\left(1-\alpha_{n}-\beta_{n}\right)\left\|\frac{x_{n+1}-x_{n}}{\alpha_{n}+\beta_{n}}\right\|^{2} \\
& =\left(1-\alpha_{n}-\beta_{n}\right)\left\|x_{n}-p\right\|^{2}+\alpha_{n}\left\|k_{n}-p\right\|^{2} \\
& +\beta_{n}\left\|W_{n} z_{n}-p\right\|^{2}-\frac{1-\alpha_{n}-\beta_{n}}{\alpha_{n}+\beta_{n}}\left\|x_{n+1}-x_{n}\right\|^{2} \\
& \leq\left(1-\alpha_{n}-\beta_{n}\right)\left\|x_{n}-p\right\|^{2}+\alpha_{n}\left[\left(1+\gamma_{n}\right)\left\|z_{n}-p\right\|^{2}+c_{n}\right] \\
& +\beta_{n}\left\|z_{n}-p\right\|^{2}-\frac{1-\alpha_{n}-\beta_{n}}{\alpha_{n}+\beta_{n}}\left\|x_{n+1}-x_{n}\right\|^{2} \\
& \leq\left(1-\alpha_{n}-\beta_{n}\right)\left\|x_{n}-p\right\|^{2}+\alpha_{n}\left[\left(1+\gamma_{n}\right)\left\|x_{n}-p\right\|^{2}+c_{n}\right] \\
& +\beta_{n}\left\|x_{n}-p\right\|^{2}-\frac{1-\alpha_{n}-\beta_{n}}{\alpha_{n}+\beta_{n}}\left\|x_{n+1}-x_{n}\right\|^{2}
\end{aligned}
$$

$$
\begin{aligned}
= & \left(1-\alpha_{n}\right)\left\|x_{n}-p\right\|^{2}+\alpha_{n}\left[\left(1+\gamma_{n}\right)\left\|x_{n}-p\right\|^{2}+c_{n}\right] \\
& -\frac{1-\alpha_{n}-\beta_{n}}{\alpha_{n}+\beta_{n}}\left\|x_{n+1}-x_{n}\right\|^{2} \\
= & \left(1+\alpha_{n} \gamma_{n}\right)\left\|x_{n}-p\right\|^{2}+\alpha_{n} c_{n}-\frac{1-\alpha_{n}-\beta_{n}}{\alpha_{n}+\beta_{n}}\left\|x_{n+1}-x_{n}\right\|^{2} \\
\leq & \left(1+\gamma_{n}\right)\left\|x_{n}-p\right\|^{2}+c_{n}-\left(1-\alpha_{n}-\beta_{n}\right)\left\|x_{n+1}-x_{n}\right\|^{2},
\end{aligned}
$$

which immediately yields

$$
\begin{gathered}
\left(1-\alpha_{n}-\beta_{n}\right)\left\|x_{n+1}-x_{n}\right\|^{2} \leq \\
+x_{n}-p\left\|^{2}-\right\| x_{n+1}-p \|^{2} \\
+\gamma_{n}\left\|x_{n}-p\right\|^{2}+c_{n} .
\end{gathered}
$$

From $\lim _{n \rightarrow \infty} \gamma_{n}=0, \lim _{n \rightarrow \infty} c_{n}=0, \limsup _{n \rightarrow \infty}\left(\alpha_{n}+\beta_{n}\right)<$ 1 , and the existence of $\lim _{n \rightarrow \infty}\left\|x_{n}-p\right\|$, it follows that

$$
\lim _{n \rightarrow \infty}\left\|x_{n+1}-x_{n}\right\|=0 \text {. }
$$

Again, utilizing Lemma 7(b), we obtain from (105), (106), and (111) that

$$
\begin{aligned}
&\left\|x_{n+1}-p\right\|^{2} \\
&=\left\|\left(1-\alpha_{n}-\beta_{n}\right)\left(x_{n}-p\right)+\alpha_{n}\left(k_{n}-p\right)+\beta_{n}\left(W_{n} z_{n}-p\right)\right\|^{2} \\
& \leq\left(1-\alpha_{n}-\beta_{n}\right)\left\|x_{n}-p\right\|^{2}+\alpha_{n}\left\|k_{n}-p\right\|^{2} \\
&+\beta_{n}\left\|W_{n} z_{n}-p\right\|^{2}-\beta_{n}\left(1-\alpha_{n}-\beta_{n}\right)\left\|W_{n} z_{n}-x_{n}\right\|^{2} \\
& \leq\left(1-\alpha_{n}-\beta_{n}\right)\left\|x_{n}-p\right\|^{2}+\alpha_{n}\left[\left(1+\gamma_{n}\right)\left\|z_{n}-p\right\|^{2}+c_{n}\right] \\
&+\beta_{n}\left\|z_{n}-p\right\|^{2}-\beta_{n}\left(1-\alpha_{n}-\beta_{n}\right)\left\|W_{n} z_{n}-x_{n}\right\|^{2} \\
& \leq\left(1-\alpha_{n}-\beta_{n}\right)\left\|x_{n}-p\right\|^{2}+\alpha_{n}\left[\left(1+\gamma_{n}\right)\left\|x_{n}-p\right\|^{2}+c_{n}\right] \\
&+\beta_{n}\left\|x_{n}-p\right\|^{2}-\beta_{n}\left(1-\alpha_{n}-\beta_{n}\right)\left\|W_{n} z_{n}-x_{n}\right\|^{2} \\
&=\left(1-\alpha_{n}\right)\left\|x_{n}-p\right\|^{2}+\alpha_{n}\left[\left(1+\gamma_{n}\right)\left\|x_{n}-p\right\|^{2}+c_{n}\right] \\
&-\beta_{n}\left(1-\alpha_{n}-\beta_{n}\right)\left\|W_{n} z_{n}-x_{n}\right\|^{2} \\
&=\left(1+\alpha_{n} \gamma_{n}\right)\left\|x_{n}-p\right\|^{2}+\alpha_{n} c_{n} \\
&-\beta_{n}\left(1-\alpha_{n}-\beta_{n}\right)\left\|W_{n} z_{n}-x_{n}\right\|^{2} \\
& \leq\left(1+\gamma_{n}\right)\left\|x_{n}-p\right\|^{2}+c_{n}-\beta_{n}\left(1-\alpha_{n}-\beta_{n}\right)\left\|W_{n} z_{n}-x_{n}\right\|^{2},
\end{aligned}
$$

which leads to

$$
\begin{aligned}
\beta_{n}\left(1-\alpha_{n}-\beta_{n}\right)\left\|W_{n} z_{n}-x_{n}\right\|^{2} \leq & \left\|x_{n}-p\right\|^{2}-\left\|x_{n+1}-p\right\|^{2} \\
& +\gamma_{n}\left\|x_{n}-p\right\|^{2}+c_{n} .
\end{aligned}
$$

From $\lim _{n \rightarrow \infty} \gamma_{n}=0, \lim _{n \rightarrow \infty} c_{n}=0$, condition (ii), and the existence of $\lim _{n \rightarrow \infty}\left\|x_{n}-p\right\|$, it follows that

$$
\lim _{n \rightarrow \infty}\left\|W_{n} z_{n}-x_{n}\right\|=0 \text {. }
$$


Note that

$$
x_{n+1}-x_{n}=\alpha_{n}\left(k_{n}-x_{n}\right)+\beta_{n}\left(W_{n} z_{n}-x_{n}\right) .
$$

Hence, it is easy to see from $0<a \leq \alpha_{n} \leq 1$ that

$$
\begin{aligned}
a\left\|x_{n}-k_{n}\right\| & \leq \alpha_{n}\left\|k_{n}-x_{n}\right\| \\
& \leq\left\|x_{n+1}-x_{n}-\beta_{n}\left(W_{n} z_{n}-x_{n}\right)\right\| \\
& \leq\left\|x_{n+1}-x_{n}\right\|+\beta_{n}\left\|W_{n} z_{n}-x_{n}\right\| \\
& \leq\left\|x_{n+1}-x_{n}\right\|+\left\|W_{n} z_{n}-x_{n}\right\| .
\end{aligned}
$$

From (115) and (118), it follows that

$$
\lim _{n \rightarrow \infty}\left\|k_{n}-x_{n}\right\|=0
$$

Combining (107) and (111), we have

$$
\begin{aligned}
\left\|k_{n}-p\right\|^{2} \leq & \left\|z_{n}-p\right\|^{2}+\gamma_{n}\left\|z_{n}-p\right\|^{2}+c_{n} \\
\leq & \left\|u_{n}-p\right\|^{2}+\gamma_{n}\left\|z_{n}-p\right\|^{2}+c_{n} \\
\leq & \left\|\Theta_{n}^{k} x_{n}-p\right\|^{2}+\gamma_{n} M_{1}+c_{n} \\
\leq & \left\|x_{n}-p\right\|^{2}+r_{k, n}\left(r_{k, n}-2 \mu_{k}\right)\left\|A_{k} \Theta_{n}^{k-1} x_{n}-A_{k} p\right\|^{2} \\
& \quad+\gamma_{n} M_{1}+c_{n},
\end{aligned}
$$

where $M_{1}=\sup _{n \geq 1}\left\|z_{n}-p\right\|^{2}$, which implies

$$
\begin{aligned}
& r_{k, n}\left(2 \mu_{k}-r_{k, n}\right)\left\|A_{k} \Theta_{n}^{k-1} x_{n}-A_{k} p\right\|^{2} \\
& \quad \leq\left\|x_{n}-p\right\|^{2}-\left\|k_{n}-p\right\|^{2}+\gamma_{n} M_{1}+c_{n} \\
& \quad \leq\left\|x_{n}-k_{n}\right\|\left(\left\|x_{n}-p\right\|+\left\|k_{n}-p\right\|\right)+\gamma_{n} M_{1}+c_{n} .
\end{aligned}
$$

From $\left\{r_{k, n}\right\} \subset\left[e_{k}, f_{k}\right] \subset\left(0,2 \mu_{k}\right), k \in\{1,2, \ldots, M\}$, $\lim _{n \rightarrow \infty} \gamma_{n}=0, \lim _{n \rightarrow \infty} c_{n}=0$, and (121), we have

$$
\lim _{n \rightarrow \infty}\left\|A_{k} \Theta_{n}^{k-1} x_{n}-A_{k} p\right\|=0, \quad k=1,2, \ldots, M
$$

Combining (108) and (111), we have

$$
\begin{aligned}
\left\|k_{n}-p\right\|^{2} \leq & \left\|\Theta_{n}^{k} x_{n}-p\right\|^{2}+\gamma_{n} M_{1}+c_{n} \\
\leq & \left\|x_{n}-p\right\|^{2}-\left\|\Theta_{n}^{k-1} x_{n}-\Theta_{n}^{k} x_{n}\right\|^{2} \\
& +2 r_{k, n}\left\|\Theta_{n}^{k-1} x_{n}-\Theta_{n}^{k} x_{n}\right\|\left\|A_{k} \Theta_{n}^{k-1} x_{n}-A_{k} p\right\| \\
& +\gamma_{n} M_{1}+c_{n},
\end{aligned}
$$

which implies

$$
\begin{aligned}
& \left\|\Theta_{n}^{k-1} x_{n}-\Theta_{n}^{k} x_{n}\right\|^{2} \\
& \leq\left\|x_{n}-p\right\|^{2}-\left\|k_{n}-p\right\|^{2} \\
& \quad+2 r_{k, n}\left\|\Theta_{n}^{k-1} x_{n}-\Theta_{n}^{k} x_{n}\right\|\left\|A_{k} \Theta_{n}^{k-1} x_{n}-A_{k} p\right\|+\gamma_{n} M_{1}+c_{n} \\
& \leq\left\|x_{n}-k_{n}\right\|\left(\left\|x_{n}-p\right\|+\left\|k_{n}-p\right\|\right) \\
& +2 r_{k, n}\left\|\Theta_{n}^{k-1} x_{n}-\Theta_{n}^{k} x_{n}\right\|\left\|A_{k} \Theta_{n}^{k-1} x_{n}-A_{k} p\right\|+\gamma_{n} M_{1}+c_{n} .
\end{aligned}
$$

From $\lim _{n \rightarrow \infty} \gamma_{n}=0, \lim _{n \rightarrow \infty} c_{n}=0$, (121), and (124), we get

$$
\lim _{n \rightarrow \infty}\left\|\Theta_{n}^{k-1} x_{n}-\Theta_{n}^{k} x_{n}\right\|=0, \quad k=1,2, \ldots, M
$$

From (127), we have

$$
\begin{aligned}
\left\|x_{n}-u_{n}\right\|= & \left\|\Theta_{n}^{0} x_{n}-\Theta_{n}^{M} x_{n}\right\| \\
\leq & \left\|\Theta_{n}^{0} x_{n}-\Theta_{n}^{1} x_{n}\right\|+\left\|\Theta_{n}^{1} x_{n}-\Theta_{n}^{2} x_{n}\right\| \\
& +\cdots+\left\|\Theta_{n}^{M-1} x_{n}-\Theta_{n}^{M} x_{n}\right\| \longrightarrow \quad \text { as } n \longrightarrow \infty .
\end{aligned}
$$

Combining (109) and (111), we obtain

$$
\begin{aligned}
\left\|k_{n}-p\right\|^{2} \leq & \left\|z_{n}-p\right\|^{2}+\gamma_{n}\left\|z_{n}-p\right\|^{2}+c_{n} \\
\leq & \left\|\Omega_{n}^{i} u_{n}-p\right\|^{2}+\gamma_{n} M_{1}+c_{n} \\
\leq & \left\|x_{n}-p\right\|^{2}+\lambda_{i, n}\left(\lambda_{i, n}-2 \eta_{i}\right)\left\|B_{i} \Omega_{n}^{i-1} u_{n}-B_{i} p\right\|^{2} \\
& +\gamma_{n} M_{1}+c_{n},
\end{aligned}
$$

where $i \in\{1,2, \ldots, N\}$, which implies

$$
\begin{aligned}
\lambda_{i, n}\left(2 \eta_{i}-\lambda_{i, n}\right)\left\|B_{i} \Omega_{n}^{i-1} u_{n}-B_{i} p\right\|^{2} \\
\leq\left\|x_{n}-p\right\|^{2}-\left\|k_{n}-p\right\|^{2}+2 \lambda_{n} \alpha_{n}\|p\|\left\|p-t_{n}\right\| \\
\quad+\gamma_{n}\left\|z_{n}-p\right\|^{2}+c_{n} \\
\leq\left\|x_{n}-k_{n}\right\|\left(\left\|x_{n}-p\right\|+\left\|k_{n}-p\right\|\right)+\gamma_{n} M_{1}+c_{n} .
\end{aligned}
$$

From $\left\{\lambda_{i, n}\right\} \subset\left[a_{i}, b_{i}\right] \subset\left(0,2 \eta_{i}\right), i \in\{1,2, \ldots, N\}, \lim _{n \rightarrow \infty} \gamma_{n}=$ $0, \lim _{n \rightarrow \infty} c_{n}=0$, and (121), we get

$$
\lim _{n \rightarrow \infty}\left\|B_{i} \Omega_{n}^{i-1} u_{n}-B_{i} p\right\|=0, \quad i \in\{1,2, \ldots, N\}
$$


Combining (110) and (111), we have

$$
\begin{aligned}
\left\|k_{n}-p\right\|^{2} \leq & \left\|z_{n}-p\right\|^{2}+\gamma_{n}\left\|z_{n}-p\right\|^{2}+c_{n} \\
\leq & \left\|\Omega_{n}^{i} u_{n}-p\right\|^{2}+\gamma_{n} M_{1}+c_{n} \\
\leq & \left\|x_{n}-p\right\|^{2}-\left\|\Omega_{n}^{i-1} u_{n}-\Omega_{n}^{i} u_{n}\right\|^{2} \\
& +2 \lambda_{i, n}\left\|\Omega_{n}^{i-1} u_{n}-\Omega_{n}^{i} u_{n}\right\|\left\|B_{i} \Omega_{n}^{i-1} u_{n}-B_{i} p\right\| \\
& +\gamma_{n} M_{1}+c_{n},
\end{aligned}
$$

which implies

$$
\begin{aligned}
& \left\|\Omega_{n}^{i-1} u_{n}-\Omega_{n}^{i} u_{n}\right\|^{2} \\
& \leq\left\|x_{n}-p\right\|^{2}-\left\|k_{n}-p\right\|^{2} \\
& \quad+2 \lambda_{i, n}\left\|\Omega_{n}^{i-1} u_{n}-\Omega_{n}^{i} u_{n}\right\|\left\|B_{i} \Omega_{n}^{i-1} u_{n}-B_{i} p\right\|+\gamma_{n} M_{1}+c_{n} \\
& \leq\left\|x_{n}-k_{n}\right\|\left(\left\|x_{n}-p\right\|+\left\|k_{n}-p\right\|\right) \\
& \quad+2 \lambda_{i, n}\left\|\Omega_{n}^{i-1} u_{n}-\Omega_{n}^{i} u_{n}\right\|\left\|B_{i} \Omega_{n}^{i-1} u_{n}-B_{i} p\right\|+\gamma_{n} M_{1}+c_{n} .
\end{aligned}
$$

From $\lim _{n \rightarrow \infty} \gamma_{n}=0, \lim _{n \rightarrow \infty} c_{n}=0$, (121), and (131), we obtain

$$
\lim _{n \rightarrow \infty}\left\|\Omega_{n}^{i-1} u_{n}-\Omega_{n}^{i} u_{n}\right\|=0, \quad i \in\{1,2, \ldots, N\} .
$$

By (134), we have

$$
\begin{aligned}
\left\|u_{n}-z_{n}\right\|= & \left\|\Omega_{n}^{0} u_{n}-\Omega_{n}^{N} u_{n}\right\| \\
\leq & \left\|\Omega_{n}^{0} u_{n}-\Omega_{n}^{1} u_{n}\right\|+\left\|\Omega_{n}^{1} u_{n}-\Omega_{n}^{2} u_{n}\right\| \\
& +\cdots+\left\|\Omega_{n}^{N-1} u_{n}-\Omega_{n}^{N} u_{n}\right\| \longrightarrow 0 \quad \text { as } n \longrightarrow \infty .
\end{aligned}
$$

From (128) and (135), we have

$$
\begin{array}{r}
\left\|x_{n}-z_{n}\right\| \leq\left\|x_{n}-u_{n}\right\|+\left\|u_{n}-z_{n}\right\| \\
\longrightarrow 0 \quad \text { as } n \longrightarrow \infty .
\end{array}
$$

By (121) and (136), we obtain

$$
\begin{array}{r}
\left\|k_{n}-z_{n}\right\| \leq\left\|k_{n}-x_{n}\right\|+\left\|x_{n}-z_{n}\right\| \\
\longrightarrow 0 \quad \text { as } n \longrightarrow \infty .
\end{array}
$$

We note that

$$
k_{n}-z_{n}=\left(1-\delta_{n}\right)\left(S^{n} z_{n}-z_{n}\right) .
$$

From $\delta_{n} \leq d<1$ and (137), we have

$$
\lim _{n \rightarrow \infty}\left\|S^{n} z_{n}-z_{n}\right\|=0 \text {. }
$$

On the other hand, we observe that

$$
\left\|z_{n+1}-z_{n}\right\| \leq\left\|z_{n+1}-x_{n+1}\right\|+\left\|x_{n+1}-x_{n}\right\|+\left\|x_{n}-z_{n}\right\| .
$$

By (115) and (136), we have

$$
\lim _{n \rightarrow \infty}\left\|z_{n+1}-z_{n}\right\|=0
$$

We note that

$$
\begin{aligned}
\left\|z_{n}-S z_{n}\right\| \leq & \left\|z_{n}-z_{n+1}\right\|+\left\|z_{n+1}-S^{n+1} z_{n+1}\right\| \\
& +\left\|S^{n+1} z_{n+1}-S^{n+1} z_{n}\right\|+\left\|S^{n+1} z_{n}-S z_{n}\right\| .
\end{aligned}
$$

From (139), (141), Lemma 9, and the uniform continuity of $S$, we obtain

$$
\lim _{n \rightarrow \infty}\left\|z_{n}-S z_{n}\right\|=0 .
$$

In addition, note that

$$
\begin{aligned}
\left\|z_{n}-W z_{n}\right\| & \leq\left\|z_{n}-W_{n} z_{n}\right\|+\left\|W_{n} z_{n}-W z_{n}\right\| \\
& \leq\left\|z_{n}-x_{n}\right\|+\left\|x_{n}-W_{n} z_{n}\right\|+\left\|W_{n} z_{n}-W z_{n}\right\| .
\end{aligned}
$$

So, from (118), (136), and [4, Remark 3.2], it follows that

$$
\lim _{n \rightarrow \infty}\left\|z_{n}-W z_{n}\right\|=0
$$

Since $\left\{x_{n}\right\}$ is bounded, there exists a subsequence $\left\{x_{n_{i}}\right\}$ of $\left\{x_{n}\right\}$ which converges weakly to $w$. From (136), we have that $z_{n_{i}} \rightarrow w$. From (143) and the uniform continuity of $S$, we have $\lim _{n \rightarrow \infty}\left\|z_{n}-S^{m} z_{n}\right\|=0$, for any $m \geq 1$. So, from Lemma 11, we have $w \in \operatorname{Fix}(S)$. Utilizing the similar arguments to those in the proof of Theorem 24, we can derive $w \in \bigcap_{k=1}^{M} \operatorname{GMEP}\left(F_{k}, \varphi_{k}, A_{k}\right) \cap \bigcap_{i=1}^{N} I\left(B_{i}, R_{i}\right) \cap \operatorname{Fix}(W)$. Consequently, $w \in F$. This shows that $\omega_{w}\left(x_{n}\right) \subset F$.

Next, let us show that $\omega_{w}\left(x_{n}\right)$ is a single-point set. As a matter of fact, let $\left\{x_{n_{j}}\right\}$ be another subsequence of $\left\{x_{n}\right\}$ such that $x_{n_{j}} \rightarrow w^{\prime}$. Then, we get $w^{\prime} \in F$. If $w \neq w^{\prime}$, from the Opial condition, we have

$$
\begin{aligned}
\lim _{n \rightarrow \infty}\left\|x_{n}-w\right\| & =\lim _{i \rightarrow \infty}\left\|x_{n_{i}}-w\right\|<\lim _{i \rightarrow \infty}\left\|x_{n_{i}}-w^{\prime}\right\| \\
& =\lim _{n \rightarrow \infty}\left\|x_{n}-w^{\prime}\right\|=\lim _{j \rightarrow \infty}\left\|x_{n_{j}}-w^{\prime}\right\| \\
& <\lim _{j \rightarrow \infty}\left\|x_{n_{j}}-w\right\|=\lim _{n \rightarrow \infty}\left\|x_{n}-w\right\| .
\end{aligned}
$$

This attains a contradiction. So we have $w=w^{\prime}$. Put $v_{n}=$ $P_{F}\left(x_{n}\right)$. Since $w \in F$, we have $\left\langle x_{n}-v_{n}, v_{n}-w\right\rangle \geq 0$. By Lemma 15, we have that $\left\{v_{n}\right\}$ converges strongly to some $w_{0} \in$ $F$. Since $\left\{x_{n}\right\}$ converges weakly to $w$, we have

$$
\left\langle w-w_{0}, w_{0}-w\right\rangle \geq 0 \text {. }
$$

Therefore, we obtain $w=w_{0}=\lim _{n \rightarrow \infty} P_{F} x_{n}$. This completes the proof. 


\section{Conflict of Interests}

The authors declare that there is no conflict of interests regarding the publication of this paper.

\section{Acknowledgments}

This project was funded by the Deanship of Scientific Research (DSR), King Abdulaziz University, under Grant no. 30-130-35-HiCi. The authors, therefore, acknowledge the technical and financial support of KAU. The authors thank the referees for their valuable comments and appreciation.

\section{References}

[1] J.-W. Peng and J.-C. Yao, "A new hybrid-extragradient method for generalized mixed equilibrium problems, fixed point problems and variational inequality problems," Taiwanese Journal of Mathematics, vol. 12, no. 6, pp. 1401-1432, 2008.

[2] L.-C. Ceng and J.-C. Yao, "A hybrid iterative scheme for mixed equilibrium problems and fixed point problems," Journal of Computational and Applied Mathematics, vol. 214, no. 1, pp. 186201, 2008.

[3] S. Takahashi and W. Takahashi, "Strong convergence theorem for a generalized equilibrium problem and a nonexpansive mapping in a Hilbert space," Nonlinear Analysis: Theory, Methods \& Applications, vol. 69, no. 3, pp. 1025-1033, 2008.

[4] Y. Yao, Y.-C. Liou, and J.-C. Yao, "Convergence theorem for equilibrium problems and fixed point problems of infinite family of nonexpansive mappings," Fixed Point Theory and Applications, vol. 2007, Article ID 064363, 12 pages, 2007.

[5] L. C. Ceng, A. Petruşel, and J. C. Yao, "Iterative approaches to solving equilibrium problems and fixed point problems of infinitely many nonexpansive mappings," Journal of Optimization Theory and Applications, vol. 143, no. 1, pp. 37-58, 2009.

[6] J. L. Lions, Quelques Méthodes de Résolution des Problèmes aux Limites non Linéaires, Dunod, Paris, France, 1969.

[7] K. Goebel and W. A. Kirk, "A fixed point theorem for asymptotically nonexpansive mappings," Proceedings of the American Mathematical Society, vol. 35, no. 1, pp. 171-174, 1972.

[8] R. Bruck, T. Kuczumow, and S. Reich, "Convergence of iterates of asymptotically nonexpansive mappings in Banach spaces with the uniform Opial property," Colloquium Mathematicum, vol. 65 , no. 2, pp. 169-179, 1993.

[9] T.-H. Kim and H.-K. Xu, "Convergence of the modified Mann's iteration method for asymptotically strict pseudo-contractions," Nonlinear Analysis: Theory, Methods \& Applications, vol. 68, no. 9, pp. 2828-2836, 2008.

[10] D. R. Sahu, H.-K. Xu, and J.-C. Yao, "Asymptotically strict pseudocontractive mappings in the intermediate sense," Nonlinear Analysis: Theory, Methods \& Applications, vol. 70, no. 10, pp. 3502-3511, 2009.

[11] N.-J. Huang, "A new completely general class of variational inclusions with noncompact valued mappings," Computers \& Mathematics with Applications, vol. 35, no. 10, pp. 9-14, 1998.

[12] L.-C. Zeng, S.-M. Guu, and J.-C. Yao, "Characterization of $H$-monotone operators with applications to variational inclusions," Computers \& Mathematics with Applications, vol. 50, no. 3-4, pp. 329-337, 2005.

[13] M. O. Osilike, S. C. Aniagbosor, and B. G. Akuchu, "Fixed points of asymptotically demicontractive mappings in arbitrary
Banach spaces," Panamerican Mathematical Journal, vol. 12, no. 2, pp. 77-88, 2002.

[14] K. K. Tan and H. K. Xu, "Approximating fixed points of nonexpansive mappings by the Ishikawa iteration process," Journal of Mathematical Analysis and Applications, vol. 178, no. 2, pp. 301-308, 1993.

[15] Z. Opial, "Weak convergence of the sequence of successive approximations for nonexpansive mappings," Bulletin of the American Mathematical Society, vol. 73, no. 4, pp. 591-597, 1967.

[16] S. Huang, "Hybrid extragradient methods for asymptotically strict pseudo-contractions in the intermediate sense and variational inequality problems," Optimization, vol. 60, no. 6, pp. 739-754, 2011.

[17] C. Martinez-Yanes and H.-K. Xu, "Strong convergence of the CQ method for fixed point iteration processes," Nonlinear Analysis: Theory, Methods \& Applications, vol. 64, no. 11, pp. 2400-2411, 2006.

[18] K. Shimoji and W. Takahashi, "Strong convergence to common fixed points of infinite nonexpansive mappings and applications," Taiwanese Journal of Mathematics, vol. 5, no. 2, pp. 387404, 2001.

[19] K. Goebel and W. A. Kirk, Topics in Metric Fixed Point Theory, Cambridge University Press, Cambridge, UK, 1990.

[20] L.-C. Ceng, Q. H. Ansari, M. M. Wong, and J.-C. Yao, "Mann type hybrid extragradient method for variational inequalities, variational inclusions and fixed point problems," Fixed Point Theory, vol. 13, no. 2, pp. 403-422, 2012.

[21] G. M. Korpelevič, "The extragradient method for finding saddle points and other problems," Ėkonomika i Matematicheskie Metody, vol. 12, no. 4, pp. 747-756, 1976.

[22] L. C. Ceng and J. C. Yao, "On the triple hierarchical variational inequalities with constraints of mixed equilibria, variational inclusions and systems of generalized equilibria," Tamkang Journal of Mathematics, vol. 45, no. 3, 2014. 


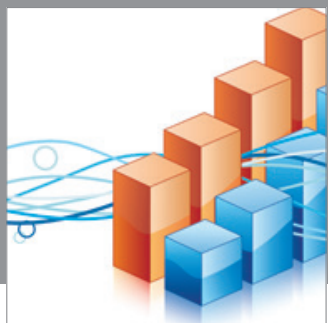

Advances in

Operations Research

mansans

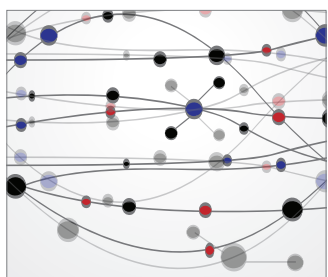

The Scientific World Journal
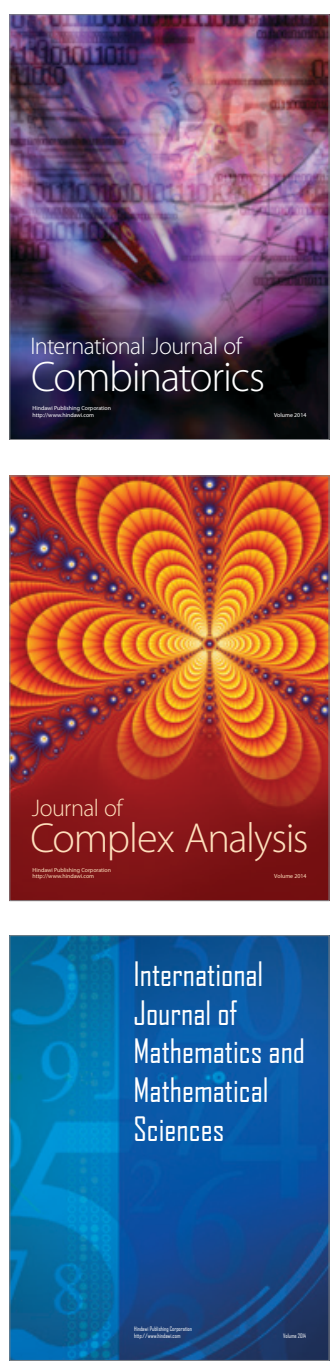
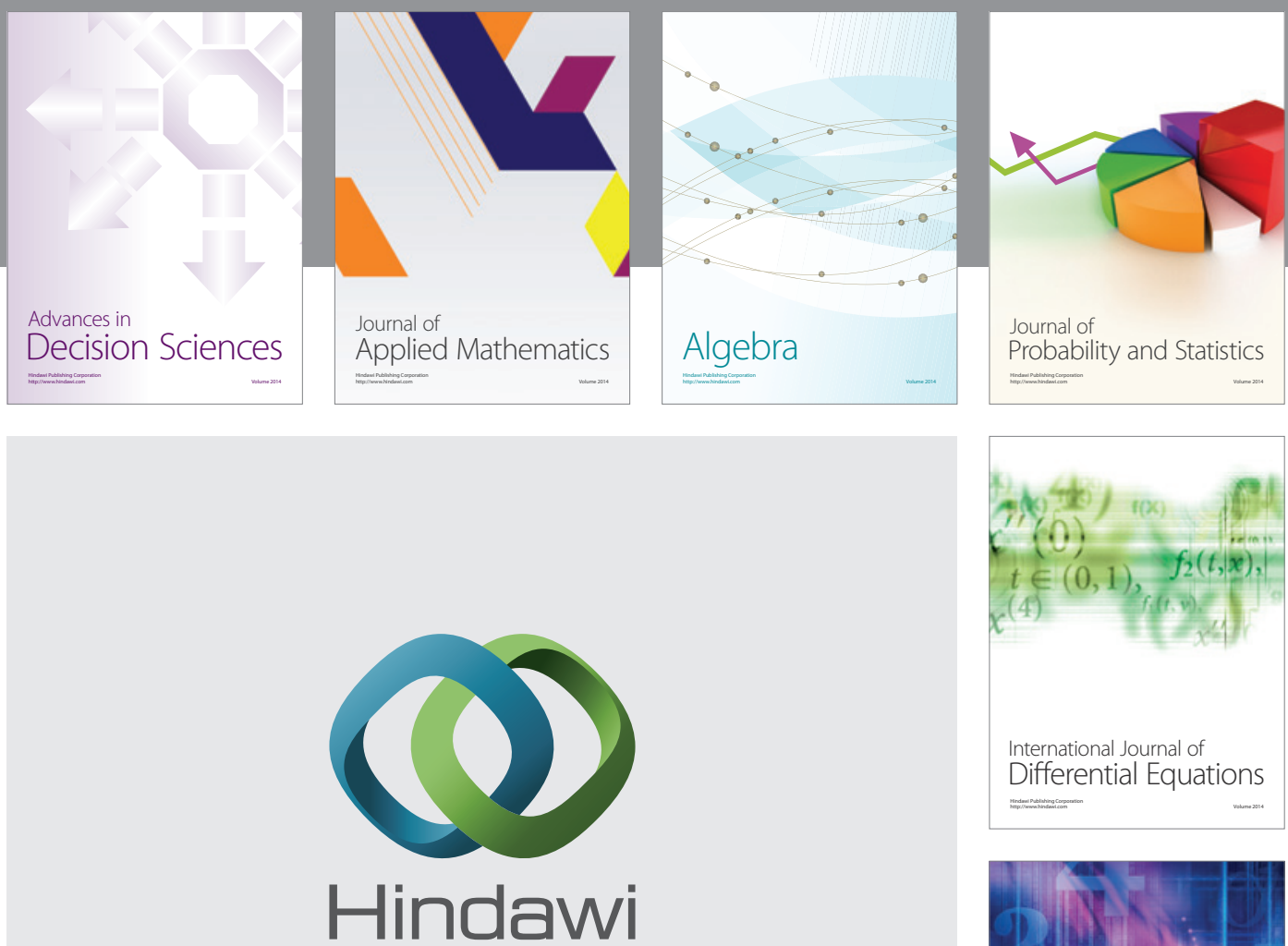

Submit your manuscripts at http://www.hindawi.com
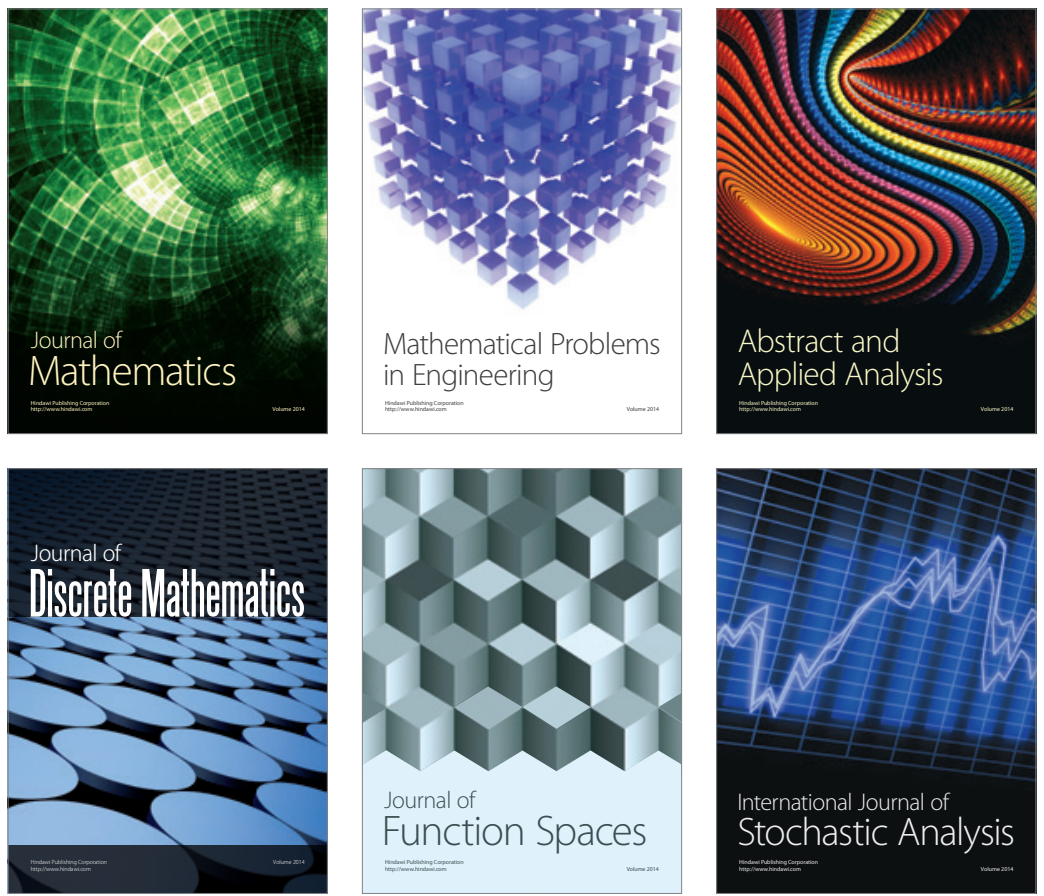

Journal of

Function Spaces

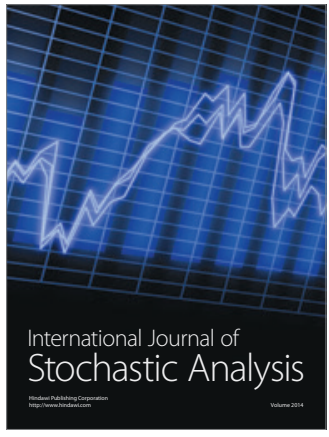

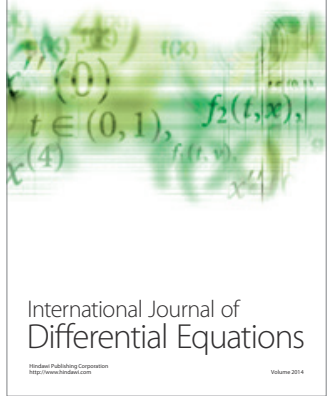
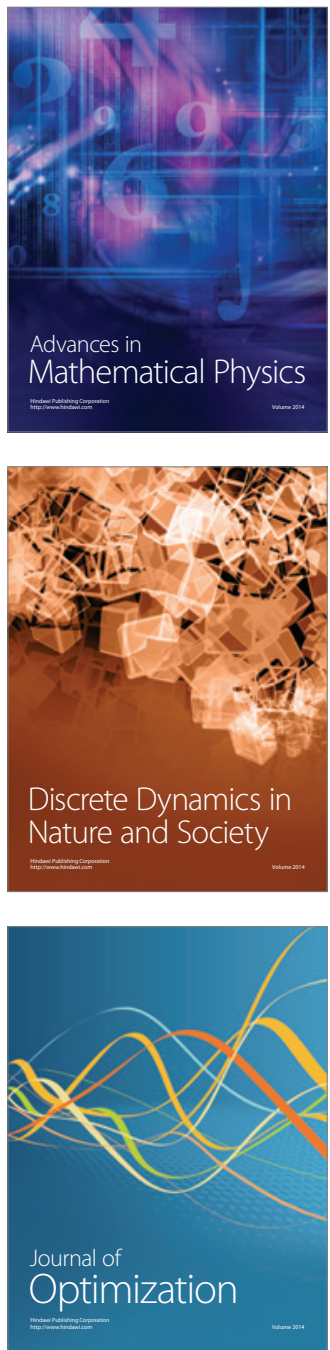